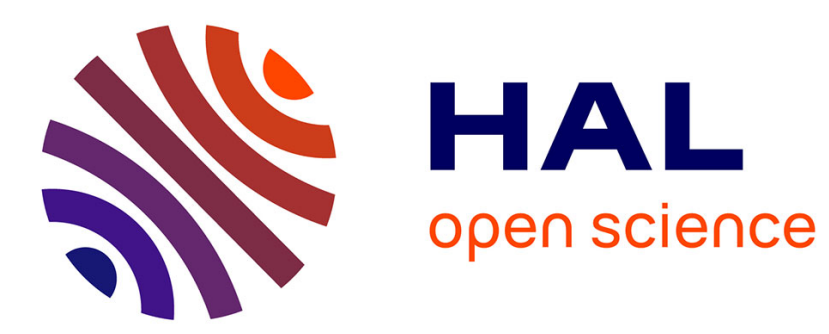

\title{
Analysis of a projection method for low-order non-conforming finite elements
}

Fanny Dardalhon, Jean-Claude Latché, Sebastian Minjeaud

\section{To cite this version:}

Fanny Dardalhon, Jean-Claude Latché, Sebastian Minjeaud. Analysis of a projection method for loworder non-conforming finite elements. IMA Journal of Numerical Analysis, 2013, 33 (1), pp. 295-317. 10.1093/imanum/drr053 . hal-00566310

\section{HAL Id: hal-00566310 https://hal.science/hal-00566310}

Submitted on 15 Feb 2011

HAL is a multi-disciplinary open access archive for the deposit and dissemination of scientific research documents, whether they are published or not. The documents may come from teaching and research institutions in France or abroad, or from public or private research centers.
L'archive ouverte pluridisciplinaire HAL, est destinée au dépôt et à la diffusion de documents scientifiques de niveau recherche, publiés ou non, émanant des établissements d'enseignement et de recherche français ou étrangers, des laboratoires publics ou privés. 


\title{
Analysis of a projection method for low-order non-conforming finite elements
}

\author{
F. Dardalhon ${ }^{1}$, J.-C. Latché ${ }^{1}$, S. Minjeaud ${ }^{2}$ \\ 1 Institut de Radioprotection et de Sûreté Nucléaire (IRSN). \\ BP3 - $13115 S^{t}$ Paul-lez-Durance cedex, France. \\ email: [fanny.dardalhon, jean-claude.latche] @irsn.fr \\ 2 Project-Team SIMPAF, \\ INRIA Lille Nord Europe Research Centre \\ Park Plazza, 40 avenue Halley, F-59650 Villeneuve d'Ascq cedex, France \\ email: sebastian.minjeaud@inria.fr
}

\begin{abstract}
We present a study of the incremental projection method to solve incompressible unsteady Stokes equations based on a low degree non-conforming finite element approximation in space, with, in particular, a piecewise constant approximation for the pressure. The numerical method falls in the class of algebraic projection methods. We provide an error analysis in the case of Dirichlet boundary conditions, which confirms that the splitting error is second-order in time. In addition, we show that pressure artificial boundary conditions are present in the discrete pressure elliptic operator, even if this operator is obtained by a splitting performed at the discrete level; however, these boundary conditions are imposed in the finite volume (weak) sense and the optimal order of approximation in space is still achieved, even for open boundary conditions.
\end{abstract}

Keywords: Incompressible flows, unsteady Stokes problem, projection methods, RannacherTurek finite elements, Crouzeix-Raviart finite elements

\section{Introduction}

We consider the time-dependent incompressible Stokes equations, posed on a finite time interval $(0, T)$ and in an open, connected, bounded domain $\Omega$ in $\mathbb{R}^{d}(d=2$, or 3$)$, which is supposed to be polygonal $(d=2)$ or polyhedral $(d=3)$ for the sake of simplicity. The system under consideration reads:

$$
\begin{array}{ll}
\partial_{t} \underline{\boldsymbol{u}}-\Delta \underline{\boldsymbol{u}}+\nabla \underline{p}=\underline{\boldsymbol{f}} & \text { in }(0, T) \times \Omega, \\
\operatorname{div} \underline{\boldsymbol{u}}=0 & \text { in }(0, T) \times \Omega,
\end{array}
$$

where $\underline{\boldsymbol{u}}$ stands for the (vector-valued) velocity, $\underline{p}$ for the (scalar) pressure, and $\boldsymbol{f}$ for a (vectorvalued) regular known forcing term. The boundary $\Gamma$ of $\Omega$ is supposed to be split in $\Gamma=$ $\Gamma_{D} \cup \Gamma_{N}$, and the measure of $\Gamma_{D}$ is assumed to be positive. The velocity is prescribed over $\Gamma_{D}$ while Neumann boundary conditions are imposed over $\Gamma_{N}$ :

$$
\begin{array}{ll}
\underline{\boldsymbol{u}}=\underline{\boldsymbol{u}}_{D} & \text { on }(0, T) \times \Gamma_{D}, \\
-\underline{p} \boldsymbol{n}+\boldsymbol{\nabla} \underline{\boldsymbol{u}} \cdot \boldsymbol{n}=\underline{\boldsymbol{f}}_{N} & \text { on }(0, T) \times \Gamma_{N} .
\end{array}
$$

This system must be supplemented by the initial condition $\underline{\boldsymbol{u}}=\underline{\boldsymbol{u}}_{0}$ on $\{0\} \times \Omega$. The vector fields $\underline{\boldsymbol{u}}_{D}, \underline{\boldsymbol{f}}_{N}$ and $\underline{\boldsymbol{u}}_{0}$ are given and supposed to be regular. 
We present in this paper a discretization of System (1.1) with non-conforming low-degree Rannacher-Turek [19] or Crouzeix-Raviart [6] finite elements. The time discretization is performed by an incremental projection method [5, 23, 9, 24]. Since the pressure is approximated by piecewise constant functions, the projection step must be left as a Darcy system. We thus choose to use a lumped discretization for the time -derivative terms, which allows us to obtain the elliptic problem for the pressure by an explicit algebraic process. Extended to variabledensity Navier-Stokes equations, this scheme is used in the open-source ISIS code [14] developed at IRSN for the computation of low-Mach-number turbulent reactive flows, and extensively used for simulation of fires.

Our results are twofold. First, we are able to lay down the scheme in a variational setting, with mesh-dependent inner-products, operators and norms, which allows us to adapt for the problem at hand the error analysis performed in the semi-discrete time setting [20,11] or for conforming elements [10]; we thus obtain, for homogeneous Dirichlet boundary conditions, a second-order estimate (with respect to the time step) for the splitting error. Second, we derive an explicit expression for the discrete elliptic operator applied to the pressure increment in the projection step. This construction brings some new elements to the rather controversial issue (in the framework of algebraic methods) of artificial pressure boundary conditions (see [12] and references therein). Indeed, we show that we obtain a finite-volume-like discretization of the Laplace operator, with the expected boundary conditions, namely homogeneous Neumann boundary conditions on $\Gamma_{D}$ and homogeneous Dirichlet boundary conditions on $\Gamma_{N}$. However, since, as usual for finite volumes, these boundary conditions are only enforced in a weak sense, their influence is observed to vanish when the time step goes to zero, and we recover optimal convergence rates with respect to the size of the mesh, even in the $L^{\infty}$ norm for the pressure in the case of open boundary conditions.

This paper is organized as follows. We first describe the scheme (Section 2), and we give the expression of the elliptic pressure operator (Section 2.3). Then we provide error bounds (Section 3), and finally, describe some numerical tests which substantiate our analysis (Section $4)$.

\section{Discretization}

This section describes the scheme under study in this paper. We first recall the principle of the standard incremental projection scheme in a time semi-discrete setting (Section 2.1) and then present its application in combination with a non-conforming finite elements space discretization (Section 2.2). Finally (Section 2.3), we give an explicit expression of the discrete operator associated to the elliptic problem satisfied by the pressure increment, and discuss the (spurious) boundary conditions which are remanent in this operator.

\subsection{The standard time-discrete projection algorithm}

Let $0=t^{0}<t^{1}<\ldots<t^{N}=T\left(N \in \mathbb{N}^{*}\right)$ define a partition of the time interval $(0, T)$, which is supposed uniform for the sake of simplicity, and let $\delta t=t^{n+1}-t^{n}$ for $n=0,1, \ldots, N-1$ be the constant time step. In a time semi-discrete setting, denoting by $\boldsymbol{u}^{0}$ the initial guess for the velocity, the usual incremental projection scheme reads, for $0 \leqslant n<N$ : 
1 - Solve for $\widetilde{\boldsymbol{u}}^{n+1}$ :

$$
\frac{1}{\delta t}\left(\widetilde{\boldsymbol{u}}^{n+1}-\boldsymbol{u}^{n}\right)-\Delta \widetilde{\boldsymbol{u}}^{n+1}+\nabla p^{n}=\boldsymbol{f}^{n+1} \quad \text { in } \Omega
$$

with the boundary conditions $\widetilde{\boldsymbol{u}}^{n+1}=\boldsymbol{u}_{D}^{n+1}$ on $\Gamma_{D}$ and $\boldsymbol{\nabla} \widetilde{\boldsymbol{u}}^{n+1} \cdot \boldsymbol{n}-p^{n} \boldsymbol{n}=\boldsymbol{f}_{N}^{n+1}$ on $\Gamma_{N}$.

2 - Solve for $p^{n+1}$ and $\boldsymbol{u}^{n+1}$ :

$$
\begin{array}{ll}
\frac{1}{\delta t}\left(\boldsymbol{u}^{n+1}-\widetilde{\boldsymbol{u}}^{n+1}\right)+\boldsymbol{\nabla}\left(p^{n+1}-p^{n}\right)=0 & \text { in } \Omega, \\
\operatorname{div} \boldsymbol{u}^{n+1}=0 & \text { in } \Omega,
\end{array}
$$

with the boundary conditions $\boldsymbol{u}^{n+1} \cdot \boldsymbol{n}=\widetilde{\boldsymbol{u}}^{n+1} \cdot \boldsymbol{n}$ on $\Gamma_{D}$ and $p^{n+1}=p^{n}$ on $\Gamma_{N}$.

Usually, for the solution of Step 2, both equations are combined (taking the divergence of the first equality and substracting to the second one) to obtain an elliptic problem for the pressure, which reads in the time semi-discrete setting:

$$
-\Delta\left(p^{n+1}-p^{n}\right)=-\frac{1}{\delta t} \operatorname{div} \widetilde{\boldsymbol{u}}^{n+1} \quad \text { in } \Omega,
$$

with the boundary conditions $\boldsymbol{\nabla}\left(p^{n+1}-p^{n}\right) \cdot \boldsymbol{n}=0$ on $\Gamma_{D}$ and $p^{n+1}=p^{n}$ on $\Gamma_{N}$.

Boundary conditions of (2.1), (2.2) and (2.3) are in a sense consistent with the strong formulation of the problem, since they enforce the fact that $\boldsymbol{u}^{n+1} \cdot \boldsymbol{n}=\boldsymbol{u}_{D}^{n+1} \cdot \boldsymbol{n}$ on $\Gamma_{D}$ and $\boldsymbol{\nabla} \widetilde{\boldsymbol{u}}^{n+1} \cdot \boldsymbol{n}-p^{n+1} \boldsymbol{n}=\boldsymbol{f}_{N}^{n+1}$ on $\Gamma_{N}$. In addition, they have for consequence that $\boldsymbol{u}^{n+1}$ is the orthogonal $\mathrm{L}^{2}$-projection of $\widetilde{\boldsymbol{u}}^{n+1}$ on:

$$
\underline{\boldsymbol{H}}=\left\{\boldsymbol{v} \in \mathrm{L}^{2}(\Omega)^{d}, \operatorname{div} \boldsymbol{v}=0, \boldsymbol{v} \cdot \boldsymbol{n}=\boldsymbol{u}_{D}^{n+1} \cdot \boldsymbol{n} \text { on } \Gamma_{D}\right\},
$$

this projection indeed being exactly the operation realized by Step 2. However, the tangential components of the end-of-step velocity do not satisfy Dirichlet boundary conditions, and spurious boundary conditions are enforced to the pressure on the whole boundary.

\subsection{The fully discrete scheme}

Let $\mathcal{M}$ be a decomposition of the domain $\Omega$ into quadrangles $(d=2)$, hexahedra $(d=3)$ or simplices $(d=2$ or $d=3)$, supposed to be regular in the usual sense of the finite element literature [8]; in particular, we exclude the presence of hanging nodes, and we suppose that the cells cannot be too flat. We denote by $\mathcal{E}$ the set of all faces $\sigma$ of the mesh, by $\mathcal{E}_{\text {ext }}$ the set of faces included in the boundary of $\Omega$, by $\mathcal{E}_{\text {int }}$ the set of internal faces $\left(i . e . \mathcal{E} \backslash \mathcal{E}_{\text {ext }}\right)$ and by $\mathcal{E}(K)$ the faces of a particular cell $K \in \mathcal{M}$. The internal face separating the neighbouring cells $K$ and $L$ is denoted by $\sigma=K \mid L$. For each cell $K \in \mathcal{M}$ and each face $\sigma \in \mathcal{E}(K), \boldsymbol{n}_{K, \sigma}$ stands for the unit normal vector to $\sigma$ outward $K$. By $|K|$ and $|\sigma|$, we denote the measure, respectively, of the control volume $K$ and of the face $\sigma$. For $K \in \mathcal{M}$, let $\boldsymbol{x}_{K}$ be an interior point of $K$, which may be chosen as the mass center of $K$. Then, for $\sigma \in \mathcal{E}(K)$, we denote by $D_{K, \sigma}$ the cone of basis $\sigma$ and of opposite vertex $\boldsymbol{x}_{K}$. For an internal face $\sigma \in \mathcal{E}_{\text {int }}, \sigma=K \mid L$, we define the dual 
cell $D_{\sigma}$ associated to $\sigma$ as $D_{\sigma}=D_{K, \sigma} \cup D_{L, \sigma}$; for an external face $\sigma \in \mathcal{E}_{\text {ext }}$ of the cell $K$, we set $D_{\sigma}=D_{K, \sigma}$. Finally, we denote by $\left|D_{\sigma}\right|$ the measure of $D_{\sigma}$.

The velocity and the pressure are discretized using the so-called Rannacher-Turek finite element [19], on quadrangles or hexahedra, and by the Crouzeix-Raviart element [6] on simplices (both being possibly combined in the same mesh). The approximation for the velocity is thus non-conforming: the discrete velocity space $\boldsymbol{V}$ is composed of functions which are discontinuous through an edge, but the jump of their integral is imposed to be zero. The degrees of freedom are located at the center of the edges of the mesh, and we choose the version of the element where they represent the average of the velocity through an edge. The set of degrees of freedom thus reads:

$$
\left\{\boldsymbol{u}_{\sigma, i}, \sigma \in \mathcal{E}, 1 \leqslant i \leqslant d\right\}
$$

We denote by $\boldsymbol{\varphi}_{\sigma}^{(i)}$ the vector shape function associated to $\boldsymbol{u}_{\sigma, i}$, which, by definition, reads $\varphi_{\sigma}^{(i)}=\varphi_{\sigma} e^{(i)}$, where $\varphi_{\sigma}$ is the Rannacher-Turek or Crouzeix-Raviart scalar shape function associated to $\sigma$ and $\boldsymbol{e}^{(i)}$ is the $i^{\text {th }}$ vector of the canonical basis of $\mathbb{R}^{d}$, and we define $\boldsymbol{u}_{\sigma}$ by $\boldsymbol{u}_{\sigma}=\sum_{i=1}^{d} \boldsymbol{u}_{\sigma, i} \boldsymbol{e}^{(i)}$. With these definitions, we have the identity:

$$
\boldsymbol{u}=\sum_{\sigma \in \mathcal{E}} \sum_{i=1}^{d} \boldsymbol{u}_{\sigma, i} \boldsymbol{\varphi}_{\sigma}^{(i)}(\boldsymbol{x})=\sum_{\sigma \in \mathcal{E}} \boldsymbol{u}_{\sigma} \varphi_{\sigma}(\boldsymbol{x}), \quad \text { for a.e. } \boldsymbol{x} \in \Omega .
$$

Let $\mathcal{E}_{D} \subset \mathcal{E}_{\text {ext }}$ be the set of edges where the velocity is prescribed. Then, as usual, these Dirichlet boundary conditions are built-in in the definition of the discrete space $\boldsymbol{V}$ :

$$
\forall \sigma \in \mathcal{E}_{D}, \text { for } 1 \leqslant i \leqslant d, 1 \leqslant n \leqslant N, \quad \boldsymbol{u}_{\sigma, i}^{n}=\frac{1}{|\sigma|} \int_{\sigma} \boldsymbol{u}_{D, i}\left(\boldsymbol{x}, t^{n}\right) \mathrm{d} \gamma,
$$

where $\boldsymbol{u}_{D, i}$ stands for the $i^{\text {th }}$ component of $\boldsymbol{u}_{D}$.

The pressure is piecewise constant, and its degrees of freedom are $\left(p_{K}\right)_{K \in \mathcal{M}}$. We denote by $Q$ the discrete pressure space.

To obtain our fractional step algorithm, as in the usual incremental scheme presented in the previous section, we split the resolution in two steps: the beginning-of-step velocity $\boldsymbol{u}^{n} \in \boldsymbol{V}$ and pressure $p^{n} \in Q$ being known, we first perform a prediction step to obtain a tentative (non divergence-free) velocity $\widetilde{\boldsymbol{u}}^{n+1} \in \boldsymbol{V}$, then we compute the end-of-step pressure $p^{n+1} \in Q$ and (divergence-free) velocity $\boldsymbol{u}^{n+1} \in \boldsymbol{V}$ in a second step. We obtain, for $0 \leqslant n<N$ :

1 - Prediction step - We seek for $\widetilde{\boldsymbol{u}}^{n+1} \in \boldsymbol{V}$ such that:

$$
\begin{aligned}
& \forall \sigma \in \mathcal{E} \backslash \mathcal{E}_{D}, \text { for } 1 \leqslant i \leqslant d \\
& \begin{aligned}
\frac{\left|D_{\sigma}\right|}{\delta t}\left[\widetilde{\boldsymbol{u}}_{\sigma, i}^{n+1}-\boldsymbol{u}_{\sigma, i}^{n}\right] & +\sum_{K \in \mathcal{M}} \int_{K} \boldsymbol{\nabla} \widetilde{\boldsymbol{u}}^{n+1}: \boldsymbol{\nabla} \boldsymbol{\varphi}_{\sigma}^{(i)} \mathrm{d} \boldsymbol{x}-\sum_{K \in \mathcal{M}} \int_{K} p^{n} \operatorname{div} \boldsymbol{\varphi}_{\sigma}^{(i)} \mathrm{d} \boldsymbol{x} \\
& =\int_{\Omega} \boldsymbol{f}\left(\boldsymbol{x}, t^{n+1}\right) \cdot \boldsymbol{\varphi}_{\sigma}^{(i)} \mathrm{d} \boldsymbol{x}+\int_{\Gamma_{N}} \boldsymbol{f}_{N}\left(\boldsymbol{x}, t^{n+1}\right) \cdot \boldsymbol{\varphi}_{\sigma}^{(i)} \mathrm{d} \gamma .
\end{aligned}
\end{aligned}
$$


2 - Projection step - We seek for $\boldsymbol{u}^{n+1} \in \boldsymbol{V}$ and $p^{n+1}$ in $Q$ such that:

$$
\begin{aligned}
& \forall \sigma \in \mathcal{E} \backslash \mathcal{E}_{D}, \text { for } 1 \leqslant i \leqslant d, \\
& \qquad \frac{\left|D_{\sigma}\right|}{\delta t}\left[\boldsymbol{u}_{\sigma, i}^{n+1}-\widetilde{\boldsymbol{u}}_{\sigma, i}^{n+1}\right]-\sum_{K \in \mathcal{M}} \int_{K}\left(p^{n+1}-p^{n}\right) \operatorname{div} \boldsymbol{\varphi}_{\sigma}^{(i)} \mathrm{d} \boldsymbol{x}=0, \\
& \forall K \in \mathcal{M}, \quad \int_{K} \operatorname{div} \boldsymbol{u}^{n+1} \mathrm{~d} \boldsymbol{x}=\sum_{\sigma \in \mathcal{E}(K)}|\sigma| \boldsymbol{u}_{\sigma}^{n+1} \cdot \boldsymbol{n}_{K, \sigma}=0 .
\end{aligned}
$$

At first glance, comparing to the semi-discrete version of the incremental projection algorithm, it may be puzzling that the whole set of Dirichlet boundary conditions (2.4) be enforced to the end-of-step velocity. In fact, the following expression of the discrete gradient $(1 \leqslant i \leqslant d$, $q \in Q)$ :

$$
\begin{array}{ll}
\forall \sigma \in \mathcal{E}_{\mathrm{int}}, \sigma=K \mid L, & \sum_{M \in \mathcal{M}} \int_{M} q \operatorname{div} \boldsymbol{\varphi}_{\sigma}^{(i)}=|\sigma|\left(q_{K}-q_{L}\right) \boldsymbol{n}_{K, \sigma}^{(i)}, \\
\forall \sigma \in \mathcal{E}_{\mathrm{ext}} \backslash \mathcal{E}_{D}, \sigma \in \mathcal{E}(K), & \sum_{M \in \mathcal{M}} \int_{M} q \operatorname{div} \boldsymbol{\varphi}_{\sigma}^{(i)}=|\sigma| q_{K} \boldsymbol{n}_{K, \sigma}^{(i)},
\end{array}
$$

shows that, for the specific discretization considered here, the discrete pressure gradient on a face $\sigma$ is colinear to its normal vector, so the velocities tangent to the faces (and thus to the boundary of the domain) are anyway left unchanged by the correction step (i.e. may them be prescribed or not).

\subsection{The discrete pressure elliptic problem ... and pressure artificial boundary conditions}

Since the discrete pressure elliptic problem is not explicitly posed (as in the time semi-discrete setting), neither are the associated boundary conditions for the pressure increment. We are going to show that these boundary conditions are however recovered when computing the discrete operator. To this purpose, let us multiply the first equation of the velocity projection step (2.5) by $\frac{1}{\left|D_{\sigma}\right|}|\sigma| \boldsymbol{n}_{K, \sigma}^{(i)}$ and sum up the equations obtained for $1 \leqslant i \leqslant d$ and $\sigma \in \mathcal{E}(K)$. We get, for any $K \in \mathcal{M}$ :

$$
\begin{aligned}
\sum_{\sigma \in \mathcal{E}_{\mathrm{int}}, \sigma=K \mid L} \frac{|\sigma|^{2}}{\left|D_{\sigma}\right|}\left[\phi_{K}^{n+1}-\phi_{L}^{n+1}\right]+\sum_{\sigma \in\left(\mathcal{E}_{\mathrm{ext}} \backslash \mathcal{E}_{D}\right) \cap \mathcal{E}(K)} & \frac{|\sigma|^{2}}{\left|D_{\sigma}\right|} \phi_{K}^{n+1} \\
= & \frac{1}{\delta t} \sum_{\sigma \in \mathcal{E}(K)}|\sigma| \widetilde{\boldsymbol{u}}_{\sigma}^{n+1} \cdot \boldsymbol{n}_{K, \sigma}
\end{aligned}
$$

where we have set $\phi_{K}^{n+1}=p_{K}^{n+1}-p_{K}^{n}$, for all $K \in \mathcal{M}$. We recognize in the left-hand side of this relation a finite-volume like approximation of the Laplace operator, however inconsistent, since, on a uniform mesh, it can easily be seen that the coefficient $|\sigma|^{2} /\left|D_{\sigma}\right|$ is $d$ times greater than in the usual finite-volume scheme; this is probably related to the fact that the RannacherTurek element is known to provide an inconsistent approximation of the Darcy problem. The expected artificial boundary conditions (i.e. those of the time semi-discrete algorithm), namely 
homogeneous Neumann boundary conditions on any $\sigma \in \mathcal{E}_{D}$ and homogeneous Dirichlet boundary conditions on any $\sigma \in \mathcal{E}_{\text {ext }} \backslash \mathcal{E}_{D}$, are built-in in this operator. However, on $\Gamma_{N}$, boundary conditions are imposed in a weaker sense than in conforming approximations where pressure degrees of freedom lie on the boundary (in this latter case, pressure increments on the boundary are, usually, set exactly to zero). We may thus expect this boundary condition to be relaxed when the time step goes to zero; this behaviour is indeed observed in the numerical experiments presented in Section 4.

\section{Discrete variational formulation and error estimates}

We suppose in this section that the velocity is prescribed to zero on the whole boundary, that is:

$$
\Gamma_{N}=\emptyset \quad \text { and } \quad \boldsymbol{u}_{\Gamma_{D}}=0 .
$$

Under this assumption, we provide an error analysis for the proposed scheme, which is performed in two steps: we first derive error estimates in an abstract discrete variational setting (Section 3.1), and then show that the present scheme enters this framework (Section 3.2).

\subsection{A formal analysis of the incremental projection method}

We give in this section the proof of the fact that the splitting error associated to the incremental projection method is second-order in time, provided that the Stokes problem is regularizing. The advantage to work with the splitting error (i.e. the difference between the solution of the projection method and the solution of the implicit scheme) is twofold. First, provided that the considered scheme may be seen as a Galerkin method, the error analysis is formally the same in the fully discrete or only time-discrete setting: we work on variational formulations, whatever the functional spaces may be discrete or continuous (in space). Second, the analysis may be performed with a backward Euler time discretization, since the splitting error is second-order for this simple scheme, as for more complicated ones as second-order BDF discretization. Of course, to obtain the convergence rate of the numerical scheme, the estimate of the splitting error must be combined with the error analysis of the implicit scheme itself, which, for the Euler scheme, is only first-order in time, and incorporates the error associated to the space discretization.

The proof presented in this section essentially consists in an adaptation of existing works: see $[20,21,22]$ for seminal works, in the time semi-discrete settings, and $[10,13,11]$ for the analysis of fully discrete (conforming) schemes. In particular, the "conforming version" of the result proven in this section is given in [11, Section 5].

We adopt a formalism derived from [10], which is here adapted to cope with possible nonconforming approximations. For $0 \leqslant n<N$, we thus suppose that the considered projection 
scheme consists in searching for $\left(\widetilde{\boldsymbol{u}}^{n+1}, \boldsymbol{u}^{n+1}, p^{n+1}\right) \in \mathcal{V} \times \mathcal{X} \times \mathcal{Q}$ such that:

$$
\begin{array}{ll}
\frac{1}{\delta t}\left(\widetilde{\boldsymbol{u}}^{n+1}-\boldsymbol{u}^{n}, \boldsymbol{v}\right)+\left\langle\boldsymbol{\nabla} \widetilde{\boldsymbol{u}}^{n+1}, \boldsymbol{\nabla} \boldsymbol{v}\right\rangle+\left(\boldsymbol{\nabla} p^{n}, \boldsymbol{v}\right)=\left\langle\boldsymbol{f}^{n+1}, \boldsymbol{v}\right\rangle, & \forall \boldsymbol{v} \in \mathcal{V}, \\
\frac{1}{\delta t}\left(\boldsymbol{u}^{n+1}-\widetilde{\boldsymbol{u}}^{n+1}, \boldsymbol{v}\right)+\left(\boldsymbol{\nabla}\left(p^{n+1}-p^{n}\right), \boldsymbol{v}\right)=0, & \forall \boldsymbol{v} \in \mathcal{X}, \\
\left(\boldsymbol{u}^{n+1}, \boldsymbol{\nabla} q\right)=0, & \forall q \in \mathcal{Q},
\end{array}
$$

where $\mathcal{V}$ stands for a discrete vector space of vector-valued functions, $\mathcal{Q}$ is a discrete vector space of scalar functions, $\mathcal{X}$ is the vector space containing the functions of $\mathcal{V}$ and the gradients of the functions of $\mathcal{Q}$, which is usually denoted by $\mathcal{X}=\mathcal{V}+\boldsymbol{\nabla} \mathcal{Q},(\cdot, \cdot)$ stands for an inner product over $\mathcal{X}$ and $\langle\cdot, \cdot\rangle$ stands for the $\mathrm{L}^{2}$-inner product. The functions of $\mathcal{V}$ and $\mathcal{Q}$ are supposed to be square-integrable, i.e. $\mathcal{V} \subset \mathrm{L}^{2}(\Omega)^{d}$ and $\mathcal{Q} \subset \mathrm{L}^{2}(\Omega)$. We define a norm over $\mathcal{X}$ by:

$$
\forall \boldsymbol{v} \in \mathcal{X}, \quad\|\boldsymbol{v}\|^{2}=(\boldsymbol{v}, \boldsymbol{v}),
$$

and we denote by $\|\cdot\|_{\mathrm{L}^{2}}$ the usual $\mathrm{L}^{2}$ norm. Two different gradient operators appear (with the same notation) in this formulation: the first one applies to the functions of $\mathcal{V}$, and its range is supposed to be included in $\mathrm{L}^{2}(\Omega)^{d \times d}$, while the second one applies to the functions of $\mathcal{Q}$ and its range is a subset of $\mathcal{X}$. The algorithm must be initialized by the data of $\boldsymbol{u}^{0} \in \mathcal{V}$ and $p^{0} \in \mathcal{Q}$.

Remark 3.1 (Definition of $\mathcal{X}, \boldsymbol{\nabla} p$ and pressure elliptic problem)

The definition of the subspace $\mathcal{X}$ is completely determined by the definition of the pressure gradient, which may be adapted to represent various pressure correction techniques. Two cases are often encountered in the literature:

- Non-algebraic methods, with pressure Poisson problem. Within this framework, in most cases, the space $\mathcal{Q}$ is included in $\mathrm{H}^{1}(\Omega), \nabla q$ represents the usual gradient of the discrete pressure $q$, and the elliptic problem for the pressure increment is obtained by an usual finite element discretization of the semi-discrete Poisson problem. Equation (3.1b) is then used twice: first with $\nabla q, q \in \mathcal{Q}$, as test functions, to obtain the pressure increment elliptic problem; second, with tests functions in $\mathcal{V}$, to obtain the orthogonal projection with respect to the $(\cdot, \cdot)$ inner product on $\mathcal{V}$ of the end-of-step velocity, which is the useful quantity for the next steps of the algorithm.

- Algebraic methods. In this case, $\mathcal{X}=\mathcal{V}$, and the operator $\boldsymbol{\nabla}$ is defined by duality (i.e. $\boldsymbol{\nabla} q$ is the representant in $\mathcal{V}$, with respect to the $(\cdot, \cdot)$ inner product, of a linear form obtained by discretization of the divergence). Note that, here also, testing with the functions $\nabla q, q \in \mathcal{Q}$ yields an elliptic problem for the pressure increment, from which the end-of-step velocity has been eliminated; the operator of this problem is the usual Schur complement of the Darcy problem (3.1b)-(3.1c). However, this is suitable in practice only if the inverse of the velocity mass matrix (i.e. the matrix associated to the $(\cdot, \cdot)$ inner product) may be easily computed.

The computations performed in the remainder of this section are valid in both cases, but, of course, the meaning of a control of $\|\nabla p\|$ depends on the considered case. The scheme studied in this paper belongs to the second class of methods, with a diagonal velocity mass matrix.

The stability of the scheme is stated in the following lemma. 
Lemma 3.1 (Stability of the SCheme) The solution of (3.1) satisfies:

$$
\begin{aligned}
\left\|\boldsymbol{u}^{n+1}\right\|^{2}+\| \widetilde{\boldsymbol{u}}^{n+1}- & \boldsymbol{u}^{n}\left\|^{2}-\right\| \boldsymbol{u}^{n}\left\|^{2}+2 \delta t\right\| \nabla \widetilde{\boldsymbol{u}}^{n+1} \|_{\mathrm{L}^{2}}^{2} \\
& +\delta t^{2}\left[\left\|\boldsymbol{\nabla} p^{n+1}\right\|^{2}-\left\|\boldsymbol{\nabla} p^{n}\right\|^{2}\right]=2 \delta t\left\langle\boldsymbol{f}^{n+1}, \widetilde{\boldsymbol{u}}^{n+1}\right\rangle .
\end{aligned}
$$

Proof. Using $\boldsymbol{v}=2 \delta t \widetilde{\boldsymbol{u}}^{n+1}$ as test function in (3.1a), we obtain:

$$
\begin{aligned}
\left\|\widetilde{\boldsymbol{u}}^{n+1}\right\|^{2}-\left\|\boldsymbol{u}^{n}\right\|^{2}+\left\|\widetilde{\boldsymbol{u}}^{n+1}-\boldsymbol{u}^{n}\right\|^{2} & +2 \delta t\left\|\boldsymbol{\nabla} \widetilde{\boldsymbol{u}}^{n+1}\right\|_{\mathrm{L}^{2}}^{2} \\
& -2 \delta t\left(\boldsymbol{\nabla} p^{n}, \widetilde{\boldsymbol{u}}^{n+1}\right)=2 \delta t\left\langle\boldsymbol{f}^{n+1}, \widetilde{\boldsymbol{u}}^{n+1}\right\rangle .
\end{aligned}
$$

Then we write (3.1b) as:

$$
\left(\left[\frac{1}{\delta t} \boldsymbol{u}^{n+1}+\boldsymbol{\nabla} p^{n+1}\right]-\left[\frac{1}{\delta t} \widetilde{\boldsymbol{u}}^{n+1}+\boldsymbol{\nabla} p^{n}\right], \boldsymbol{v}\right)=0,
$$

and choose $\boldsymbol{v}$ such that:

$$
\frac{1}{\delta t^{2}} \boldsymbol{v}=\left[\frac{1}{\delta t} \boldsymbol{u}^{n+1}+\nabla p^{n+1}\right]+\left[\frac{1}{\delta t} \widetilde{\boldsymbol{u}}^{n+1}+\boldsymbol{\nabla} p^{n}\right] \in \mathcal{X}
$$

in this equation. This yields, remarking that $\left(\boldsymbol{u}^{n+1}, \boldsymbol{\nabla} p^{n+1}\right)=0$ thanks to (3.1c):

$$
\left\|\boldsymbol{u}^{n+1}\right\|^{2}-\left\|\widetilde{\boldsymbol{u}}^{n+1}\right\|^{2}+\delta t^{2}\left[\left\|\boldsymbol{\nabla} p^{n+1}\right\|^{2}-\left\|\boldsymbol{\nabla} p^{n}\right\|^{2}\right]+2 \delta t\left(\widetilde{\boldsymbol{u}}^{n+1}, \boldsymbol{\nabla} p^{n}\right)=0 .
$$

We conclude the proof by adding Equations (3.2) and (3.3).

REMARK 3.2 Of course, Lemma 3.1 yields a control of the solution only if the right-hand side may be absorbed in the left-hand one. This is the case, with the same estimate as at the continuous level, if a discrete Poincaré inequality (Relation (3.15) below) holds.

We now introduce the implicit scheme, which consists, for $0 \leqslant n<N$ and supposing that $\overline{\boldsymbol{u}}^{n} \in \mathcal{V}$ is known, in searching for $\left(\overline{\boldsymbol{u}}^{n+1}, \bar{p}^{n+1}\right) \in \mathcal{V} \times \mathcal{Q}$ such that:

$$
\begin{array}{ll}
\frac{1}{\delta t}\left(\overline{\boldsymbol{u}}^{n+1}-\overline{\boldsymbol{u}}^{n}, \boldsymbol{v}\right)+\left\langle\boldsymbol{\nabla} \overline{\boldsymbol{u}}^{n+1}, \boldsymbol{\nabla} \boldsymbol{v}\right\rangle+\left(\boldsymbol{\nabla} \bar{p}^{n+1}, \boldsymbol{v}\right)=\left\langle\boldsymbol{f}^{n+1}, \boldsymbol{v}\right\rangle, & \forall \boldsymbol{v} \in \mathcal{V}, \\
\left(\overline{\boldsymbol{u}}^{n+1}, \boldsymbol{\nabla} q\right)=0, & \forall q \in \mathcal{Q}
\end{array}
$$

and we make the following regularity assumptions for this system:

$$
\left\|\boldsymbol{\nabla}\left(\bar{p}^{n+1}-\bar{p}^{n}\right)\right\| \leqslant c \delta t, \quad \text { for } 0 \leqslant n<N,
$$

where $c$ is a positive real number.

Let the splitting errors $\widetilde{\boldsymbol{e}}^{n} \in \mathcal{V}, \boldsymbol{e}^{n} \in \mathcal{X}$ and $\epsilon^{n} \in \mathcal{Q}$, and the quantity $\psi^{n} \in \mathcal{Q}$ be defined, for $0 \leqslant n \leqslant N$ by:

$$
\boldsymbol{e}^{n}=\boldsymbol{u}^{n}-\overline{\boldsymbol{u}}^{n}, \quad \widetilde{\boldsymbol{e}}^{n}=\widetilde{\boldsymbol{u}}^{n}-\overline{\boldsymbol{u}}^{n}, \quad \epsilon^{n}=p^{n}-\bar{p}^{n}, \quad \psi^{n}=p^{n}-\bar{p}^{n+1} .
$$


It is natural to suppose that the scheme is initialized in such a way that $\boldsymbol{e}^{0}=0$. The quantity $\bar{p}^{n}$ does not appear in the implicit scheme, but we are lead in the following to use Assumption (3.5) for $n=0$, so the issue of the estimation of $\epsilon^{0}$ indeed arises; for the sake of simplicity, and also to avoid the possible (time-)irregularities of the solution of the implicit scheme at $t=0$, we suppose that the projection algorithm is initialized with some (at least one) steps of the implicit scheme, so we also have $\epsilon^{0}=0$. Then, taking the difference between (3.1a) and (3.4a) for the first equation, between (3.1c) and (3.4b) for the third one and substracting $\overline{\boldsymbol{u}}^{n+1}$ (resp. $\bar{p}^{n+1}$ ) at the first two terms (resp. the third and fourth terms) of (3.1b), we obtain that the splitting errors are governed by the following system, for $0 \leqslant n<N$ :

$$
\begin{array}{ll}
\frac{1}{\delta t}\left(\widetilde{\boldsymbol{e}}^{n+1}-\boldsymbol{e}^{n}, \boldsymbol{v}\right)+\left\langle\nabla \widetilde{\boldsymbol{e}}^{n+1}, \boldsymbol{\nabla} \boldsymbol{v}\right\rangle+\left(\boldsymbol{\nabla} \psi^{n}, \boldsymbol{v}\right)=0, & \forall \boldsymbol{v} \in \mathcal{V}, \\
\frac{1}{\delta t}\left(\boldsymbol{e}^{n+1}-\widetilde{\boldsymbol{e}}^{n+1}, \boldsymbol{v}\right)+\left(\boldsymbol{\nabla}\left(\epsilon^{n+1}-\psi^{n}\right), \boldsymbol{v}\right)=0, & \forall \boldsymbol{v} \in \mathcal{X}, \\
\left(\boldsymbol{e}^{n+1}, \boldsymbol{\nabla} q\right)=0, & \forall q \in \mathcal{Q} .
\end{array}
$$

We now give a first estimate of the splitting errors.

LEMma 3.2 (FIRST ORDER ESTIMATES FOR THE VELOCITY)

Let us suppose that (3.5) is satisfied. Then the following estimate holds for $1 \leqslant n \leqslant N$ :

$$
\left\|\boldsymbol{e}^{n}\right\|^{2}+\sum_{k=1}^{n}\left\|\widetilde{\boldsymbol{e}}^{k}-\boldsymbol{e}^{k-1}\right\|^{2}+2 \delta t \sum_{k=1}^{n}\left\|\boldsymbol{\nabla} \widetilde{\boldsymbol{e}}^{k}\right\|_{\mathrm{L}^{2}}^{2}+\delta t^{2}\left\|\boldsymbol{\nabla} \epsilon^{n}\right\| \leqslant c \delta t^{2} .
$$

Proof. We remark that System (3.6) has the same structure as System (3.1), so Lemma 3.1 yields, for $0 \leqslant n<N$ :

$$
\left\|\boldsymbol{e}^{n+1}\right\|^{2}-\left\|\boldsymbol{e}^{n}\right\|^{2}+\left\|\widetilde{\boldsymbol{e}}^{n+1}-\boldsymbol{e}^{n}\right\|^{2}+2 \delta t\left\|\nabla \widetilde{\boldsymbol{e}}^{n+1}\right\|_{\mathrm{L}^{2}}^{2}+\delta t^{2}\left[\left\|\boldsymbol{\nabla} \epsilon^{n+1}\right\|^{2}-\left\|\boldsymbol{\nabla} \psi^{n}\right\|^{2}\right]=0 .
$$

We now observe that $\psi^{n}=\epsilon^{n}-\delta \bar{p}^{n}$ with $\delta \bar{p}^{n}=\bar{p}^{n+1}-\bar{p}^{n}$. For any $\alpha>0$, we have:

$$
\left\|\boldsymbol{\nabla} \epsilon^{n}+\boldsymbol{\nabla} \delta \bar{p}^{n}\right\|^{2} \leqslant\left(\left\|\boldsymbol{\nabla} \epsilon^{n}\right\|+\left\|\boldsymbol{\nabla} \delta \bar{p}^{n}\right\|\right)^{2} \leqslant(1+\alpha)\left\|\boldsymbol{\nabla} \epsilon^{n}\right\|^{2}+\left(1+\frac{1}{\alpha}\right)\left\|\boldsymbol{\nabla} \delta \bar{p}^{n}\right\|^{2} .
$$

We thus may write, using Assumption (3.5):

$$
\left\|\boldsymbol{\nabla} \psi^{n}\right\|^{2} \leqslant(1+\delta t)\left\|\boldsymbol{\nabla} \epsilon^{n}\right\|^{2}+\left(1+\frac{1}{\delta t}\right)\left\|\boldsymbol{\nabla} \delta \bar{p}^{n}\right\|^{2} \leqslant(1+\delta t)\left\|\boldsymbol{\nabla} \epsilon^{n}\right\|^{2}+c \delta t .
$$

Returning to (3.7), we have:

$$
\begin{aligned}
\left\|\boldsymbol{e}^{n+1}\right\|^{2}-\left\|\boldsymbol{e}^{n}\right\|^{2}+\left\|\widetilde{\boldsymbol{e}}^{n+1}-\boldsymbol{e}^{n}\right\|^{2}+2 \delta t\left\|\boldsymbol{\nabla} \widetilde{\boldsymbol{e}}^{n+1}\right\|_{\mathrm{L}^{2}}^{2} \\
+\delta t^{2}\left[\left\|\boldsymbol{\nabla} \epsilon^{n+1}\right\|^{2}-\left\|\boldsymbol{\nabla} \epsilon^{n}\right\|^{2}\right] \leqslant \delta t^{2}\left\|\boldsymbol{\nabla} \epsilon^{n}\right\|^{2}+c \delta t^{3} .
\end{aligned}
$$

Summing this equation over the time steps yields for $0<n \leqslant N$ :

$$
\begin{aligned}
\left\|\boldsymbol{e}^{n}\right\|^{2}+\sum_{k=1}^{n}\left\|\widetilde{\boldsymbol{e}}^{k}-\boldsymbol{e}^{k-1}\right\|^{2}+2 \delta t \sum_{k=1}^{n}\left\|\nabla \widetilde{\boldsymbol{e}}^{k}\right\|_{\mathrm{L}^{2}}^{2}+\delta t^{2}\left\|\nabla \epsilon^{n}\right\|^{2} \\
\leqslant \delta t \sum_{k=0}^{n-1} \delta t^{2}\left\|\boldsymbol{\nabla} \epsilon^{k}\right\|^{2}+c T \delta t^{2} .
\end{aligned}
$$


This relation yields in particular:

$$
\delta t^{2}\left\|\boldsymbol{\nabla} \epsilon^{n}\right\|^{2} \leqslant \delta t \sum_{k=0}^{n-1} \delta t^{2}\left\|\nabla \epsilon^{k}\right\|^{2}+c T \delta t^{2},
$$

and, since we suppose $\epsilon^{0}=0$, the discrete Gronwall lemma 3.3 below yields that $\delta t^{2}\left\|\nabla \epsilon^{n}\right\|^{2} \leqslant$ $c \delta t^{2}$, for $1 \leqslant n \leqslant N$. Using this inequality in (3.8) yields the conclusion.

In the course of the previous proof, we used the following discrete Gronwall lemma [18, p. $14]$.

Lemma 3.3 (Discrete Gronwall lemma) We assume that $\left(\delta^{n}\right)_{n \in \mathbb{N}}$ and $\left(g^{n}\right)_{n \in \mathbb{N}}$ are two non-negative sequences, and that the sequence $\left(\theta^{n}\right)_{n \in \mathbb{N}}$ satisfies $\theta^{0} \leqslant g^{0}$ and:

$$
\forall n \geqslant 1, \quad \theta^{n} \leqslant \sum_{k=0}^{n} g^{k}+\sum_{k=0}^{n-1} \delta^{k} \theta^{k} .
$$

Then, we have:

$$
\forall n \geqslant 1, \quad \theta^{n} \leqslant\left[\sum_{k=0}^{n} g^{k}\right] \exp \left[\sum_{k=0}^{n-1} \delta^{k}\right] .
$$

Remark 3.3 (A milder REgularity Assumption) Assumption (3.5) is the discrete counterpart of an estimate for the time-derivative of the pressure solution to the implicit scheme in $\mathrm{L}^{\infty}\left(0, T ; \mathrm{H}^{1}(\Omega)\right)$. Examining the proof of Lemma 3.2 and the statement of the discrete Gronwall lemma, it appears that a control in discrete $\mathrm{L}^{2}\left(0, T ; \mathrm{H}^{1}(\Omega)\right)$ norm would be sufficient, i.e.:

$$
\sum_{n=0}^{N-1} \delta t\left\|\nabla\left(\bar{p}^{n+1}-\bar{p}^{n}\right)\right\|^{2} \leqslant c \delta t^{2} .
$$

We now turn to the second order estimates. In this direction, the first intuition consists in supposing that the difference between the predicted and end-of-step velocity $\left\|\boldsymbol{u}^{n}-\widetilde{\boldsymbol{u}}^{n}\right\|$ (or, equivalently, $\left.\left\|\boldsymbol{e}^{n}-\widetilde{\boldsymbol{e}}^{n}\right\|\right)$ behaves as $\delta t^{2}$. This is suggested by Equation (3.1b), which may be written as:

$$
\begin{aligned}
\left(\boldsymbol{u}^{n+1}-\widetilde{\boldsymbol{u}}^{n+1}, \boldsymbol{v}\right) & =\delta t\left(\boldsymbol{\nabla}\left(p^{n+1}-p^{n}\right), \boldsymbol{v}\right) \\
& =\delta t\left(\boldsymbol{\nabla}\left(\bar{p}^{n+1}-\bar{p}^{n}\right), \boldsymbol{v}\right)+\delta t\left(\boldsymbol{\nabla}\left(\epsilon^{n+1}-\epsilon^{n}\right), \boldsymbol{v}\right), \quad \forall \boldsymbol{v} \in \mathcal{X},
\end{aligned}
$$

together with Assumption (3.5), i.e. $\left\|\boldsymbol{\nabla}\left(\bar{p}^{n+1}-\bar{p}^{n}\right)\right\| \leqslant c \delta$. Of course, it remains to show that a similar estimate holds for $\left\|\nabla\left(\epsilon^{n+1}-\epsilon^{n}\right)\right\|$; let us postpone this proof for a while (namely, to Lemma 3.5), and suppose that we have, for $0 \leqslant n \leqslant N$ :

$$
\left\|\boldsymbol{u}^{n}-\widetilde{\boldsymbol{u}}^{n}\right\|=\left\|\boldsymbol{e}^{n}-\widetilde{\boldsymbol{e}}^{n}\right\| \leqslant c \delta t^{2},
$$

where $c$ is a given real number only depending on the regularity of the solution to the implicit scheme. This relation suggests that the predicted velocity is almost (i.e. up to a second order in time error) divergence-free. The way to exploit this remark is then to reconstitute a (variational) 
momentum balance equation for $\widetilde{\boldsymbol{e}}$ and to choose as test function a projection of $\widetilde{\boldsymbol{e}}$ on the space of divergence-free functions, hence eliminating the pressure errors from the computation. To undertake this program, we need to suppose that we can build such a projection enjoying strong stability properties, which we now define.

Definition 3.1 (Inverse Stokes operator) Let $S^{-1}$ be the operator which, to any function $\boldsymbol{v} \in \mathcal{V}$ associates $S^{-1} \boldsymbol{v} \in \mathcal{V}$ defined by:

$$
\begin{array}{ll}
\left\langle\boldsymbol{\nabla} S^{-1} \boldsymbol{v}, \boldsymbol{\nabla} \boldsymbol{w}\right\rangle+(\boldsymbol{\nabla} \zeta, \boldsymbol{w})=(\boldsymbol{v}, \boldsymbol{w}), & \forall \boldsymbol{w} \in \mathcal{V}, \\
\left(\boldsymbol{\nabla} q, S^{-1} \boldsymbol{v}\right)=0, & \forall q \in \mathcal{Q},
\end{array}
$$

with $\zeta \in \mathcal{Q}$.

Then we suppose that the operator $S^{-1}$ satisfies the following properties.

(S1) The bilinear form on $\mathcal{V} \times \mathcal{V}$ defined by $(\boldsymbol{v}, \boldsymbol{w}) \rightarrow\left(S^{-1} \boldsymbol{v}, \boldsymbol{w}\right)$ is symmetric, positive and associated to a semi-norm on $\mathcal{V}$ denoted by $\|\cdot\|_{*}\left(\right.$ i.e. $\left.\|\boldsymbol{v}\|_{*}^{2}=\left(S^{-1} \boldsymbol{v}, \boldsymbol{v}\right), \forall \boldsymbol{v} \in \mathcal{V}\right)$.

(S2) In addition, for any $\boldsymbol{v} \in \mathcal{V}$, the quantity $\left\langle\nabla S^{-1} \boldsymbol{v}, \boldsymbol{\nabla} \boldsymbol{v}\right\rangle$ almost controls the (square of the) $\mathrm{L}^{2}$-norm of $\boldsymbol{v}$ :

$$
\begin{aligned}
& \forall \alpha \in(0,1), \text { there exists } c(\alpha)>0 \text { such that, } \forall \boldsymbol{v} \in \mathcal{V}, \\
& \qquad\left\langle\nabla S^{-1} \boldsymbol{v}, \boldsymbol{\nabla} \boldsymbol{v}\right\rangle \geqslant(1-\alpha)\|\boldsymbol{v}\|^{2}-c(\alpha) \inf _{\boldsymbol{w} \in \mathcal{H}}\|\boldsymbol{v}-\boldsymbol{w}\|^{2},
\end{aligned}
$$

where $\mathcal{H}$ stands for the space of divergence-free functions:

$$
\mathcal{H}=\{\boldsymbol{w} \in \mathcal{X} \text { such that }(\boldsymbol{w}, \boldsymbol{\nabla} \zeta)=0, \forall \zeta \in \mathcal{Q}\} .
$$

We show in Section 3.2 that assumption (S2) is satisfied if the continuous (stationary) Stokes problem is regularizing, which is satisfied if the domain $\Omega$ is convex [4, 2].

We are now in position to state the following control of the splitting errors.

LEMMA 3.4 (SECOND ORDER ESTIMATES FOR THE VELOCiTy)

Let us suppose that Inequality (3.9) holds. Then, under Assumptions (S1) and (S2) for the inverse Stokes operator, there exists a real number $c$ only depending on the regularity of the solution to the implicit scheme such that:

$$
\sum_{n=0}^{N} \delta t\left\|\widetilde{\boldsymbol{e}}^{n}\right\|^{2}+\sum_{n=0}^{N} \delta t\left\|\boldsymbol{e}^{n}\right\|^{2} \leqslant c \delta t^{4} .
$$

Proof. As announced, taking the difference between (3.6a) and (3.6b) written at the previous time step, we reconstitute a momentum balance equation for the predicted velocity, which reads, for $0 \leqslant n<N$ :

$$
\frac{1}{\delta t}\left(\widetilde{\boldsymbol{e}}^{n+1}-\widetilde{\boldsymbol{e}}^{n}, \boldsymbol{v}\right)+\left\langle\boldsymbol{\nabla} \widetilde{\boldsymbol{e}}^{n+1}, \boldsymbol{\nabla} \boldsymbol{v}\right\rangle-\left(\boldsymbol{\nabla} \zeta^{n}, \boldsymbol{v}\right)=0,
$$

where we have arbitrarily set $\widetilde{\boldsymbol{e}}^{0}=0$ (which is possible since $\widetilde{\boldsymbol{u}}^{0}$ never appears in the algorithm) and $\zeta^{n}$ stands for a function of $\mathcal{Q}$. Taking $\boldsymbol{v}=2 \delta t S^{-1} \widetilde{\boldsymbol{e}}^{n+1}$ in this relation and invoking Assumption (S1), we get:

$$
\left\|\widetilde{\boldsymbol{e}}^{n+1}\right\|_{*}^{2}+\left\|\widetilde{\boldsymbol{e}}^{n+1}-\widetilde{\boldsymbol{e}}^{n}\right\|_{*}^{2}-\left\|\widetilde{\boldsymbol{e}}^{n}\right\|_{*}^{2}+2 \delta t\left\langle\nabla \widetilde{\boldsymbol{e}}^{n+1}, \boldsymbol{\nabla} S^{-1} \widetilde{\boldsymbol{e}}^{n+1}\right\rangle=0 .
$$


We now apply (S2) with $\alpha=1 / 2$, which yields:

$$
\left\|\widetilde{\boldsymbol{e}}^{n+1}\right\|_{*}^{2}+\left\|\widetilde{\boldsymbol{e}}^{n+1}-\widetilde{\boldsymbol{e}}^{n}\right\|_{*}^{2}-\left\|\widetilde{\boldsymbol{e}}^{n}\right\|_{*}^{2}+\delta t\left\|\widetilde{\boldsymbol{e}}^{n+1}\right\|^{2} \leqslant 2 c(1 / 2) \delta t \inf _{\boldsymbol{w} \in \mathcal{H}}\left\|\widetilde{\boldsymbol{e}}^{n+1}-\boldsymbol{w}\right\|^{2},
$$

hence, specializing $\boldsymbol{w}$ to $\boldsymbol{e}^{n+1}$ and using (3.9):

$$
\left\|\widetilde{\boldsymbol{e}}^{n+1}\right\|_{*}^{2}+\left\|\widetilde{\boldsymbol{e}}^{n+1}-\widetilde{\boldsymbol{e}}^{n}\right\|_{*}^{2}-\left\|\widetilde{\boldsymbol{e}}^{n}\right\|_{*}^{2}+\delta t\left\|\widetilde{\boldsymbol{e}}^{n+1}\right\|^{2} \leqslant 2 c(1 / 2) \delta t\left\|\widetilde{\boldsymbol{e}}^{n+1}-\boldsymbol{e}^{n+1}\right\|^{2} \leqslant 2 c(1 / 2) \delta t^{5} .
$$

Summing this relation for $0 \leqslant n<N$ and using $\widetilde{\boldsymbol{e}}^{0}=0$ yields the first part of the desired inequality. The second one follows by the Triangle inequality $\left\|\boldsymbol{e}^{n}\right\| \leqslant\left\|\widetilde{\boldsymbol{e}}^{n}\right\|+\left\|\widetilde{\boldsymbol{e}}^{n}-\boldsymbol{e}^{n}\right\|$ and using (3.9) once again.

We now have to prove (3.9). To this purpose, the idea is to use a proof similar to Lemma 3.2 , but for the time-increments of the errors. We thus define, for $0<n \leqslant N$ :

$$
\delta \boldsymbol{e}^{n}=\boldsymbol{e}^{n}-\boldsymbol{e}^{n-1}, \quad \delta \widetilde{\boldsymbol{e}}^{n}=\widetilde{\boldsymbol{e}}^{n}-\widetilde{\boldsymbol{e}}^{n-1}, \quad \delta \epsilon^{n}=\epsilon^{n}-\epsilon^{n-1}, \quad \delta \psi^{n}=\psi^{n}-\psi^{n-1} .
$$

Taking the difference of System (3.6) at two consecutive time steps, we obtain the following system of equations, valid for $0<n<N$ :

$$
\begin{array}{ll}
\frac{1}{\delta t}\left(\delta \widetilde{\boldsymbol{e}}^{n+1}-\delta \boldsymbol{e}^{n}, \boldsymbol{v}\right)+\left\langle\boldsymbol{\nabla} \delta \widetilde{\boldsymbol{e}}^{n+1}, \boldsymbol{\nabla} \boldsymbol{v}\right\rangle+\left(\boldsymbol{\nabla} \delta \psi^{n}, \boldsymbol{v}\right)=0, & \forall \boldsymbol{v} \in \mathcal{V}, \\
\frac{1}{\delta t}\left(\delta \boldsymbol{e}^{n+1}-\delta \widetilde{\boldsymbol{e}}^{n+1}, \boldsymbol{v}\right)+\left(\boldsymbol{\nabla}\left(\delta \epsilon^{n+1}-\delta \psi^{n}\right), \boldsymbol{v}\right)=0, & \forall \boldsymbol{v} \in \mathcal{X}, \\
\left(\delta \boldsymbol{e}^{n+1}, \boldsymbol{\nabla} q\right)=0, & \forall q \in \mathcal{Q} .
\end{array}
$$

We now make the following regularity assumptions for the implicit scheme:

$$
\left\|\nabla\left(\bar{p}^{n+1}-2 \bar{p}^{n}+p^{n-1}\right)\right\| \leqslant c \delta t^{2}, \quad \text { for } 0<n<N,
$$

where $c$ is a positive real number.

Lemma 3.5 (ERror increment Estimates) Since $\widetilde{\boldsymbol{u}}^{0}$ never appears in the algorithm, we can arbitrarily define $\widetilde{\boldsymbol{e}}^{0}=0$, so $\delta \widetilde{\boldsymbol{e}}^{1}=\widetilde{\boldsymbol{e}}^{1}$. With this notation, under Assumption (3.11), the following estimate holds for $0<n \leqslant N$ :

$$
\left\|\delta \boldsymbol{e}^{n}\right\|^{2}+2 \delta t \sum_{k=1}^{n}\left\|\boldsymbol{\nabla} \delta \widetilde{\boldsymbol{e}}^{k}\right\|_{\mathrm{L}^{2}}^{2}+\delta t^{2}\left\|\nabla \delta \epsilon^{n}\right\|^{2} \leqslant c \delta t^{4} .
$$

Consequently, $\left\|\nabla \delta \epsilon^{n}\right\| \leqslant c \delta t$ for $0<n \leqslant N$, and (3.9) holds.

Proof. The proof follows the same line as for Lemma 3.2, the equation (3.7) being now replaced by the following relation, valid for $0<n<N$ :

$$
\begin{aligned}
\left\|\delta \boldsymbol{e}^{n+1}\right\|^{2}-\left\|\delta \boldsymbol{e}^{n}\right\|^{2}+\| \delta \widetilde{\boldsymbol{e}}^{n+1}- & \delta \boldsymbol{e}^{n}\left\|^{2}+2 \delta t\right\| \boldsymbol{\nabla} \delta \widetilde{\boldsymbol{e}}^{n+1} \|_{\mathrm{L}^{2}}^{2} \\
& +\delta t^{2}\left[\left\|\boldsymbol{\nabla} \delta \epsilon^{n+1}\right\|^{2}-\left\|\boldsymbol{\nabla} \delta \psi^{n}\right\|^{2}\right]=0 .
\end{aligned}
$$


We now notice that $\delta \psi^{n}=\delta \epsilon^{n}-\left(\bar{p}^{n+1}-2 \bar{p}^{n}+\bar{p}^{n-1}\right)$, so Assumption (3.11) now yields:

$$
\left\|\boldsymbol{\nabla} \delta \psi^{n}\right\|^{2} \leqslant(1+\delta t)\left\|\boldsymbol{\nabla} \delta \epsilon^{n}\right\|^{2}+c \delta t^{3},
$$

where $c$ only depends on the regularity of the solution to the implicit scheme. Returning to (3.12), we have:

$$
\begin{aligned}
\left\|\delta \boldsymbol{e}^{n+1}\right\|^{2}-\left\|\delta \boldsymbol{e}^{n}\right\|^{2}+\| & \delta \widetilde{\boldsymbol{e}}^{n+1}-\delta \boldsymbol{e}^{n}\left\|^{2}+2 \delta t\right\| \boldsymbol{\nabla} \delta \widetilde{\boldsymbol{e}}^{n+1} \|_{\mathrm{L}^{2}}^{2} \\
& +\delta t^{2}\left[\left\|\boldsymbol{\nabla} \delta \epsilon^{n+1}\right\|^{2}-\left\|\boldsymbol{\nabla} \delta \epsilon^{n}\right\|^{2}\right] \leqslant \delta t^{3}\left\|\boldsymbol{\nabla} \delta \epsilon^{n}\right\|^{2}+c \delta t^{5} .
\end{aligned}
$$

Summing this equation over the time steps yields for $1<n \leqslant N$ :

$$
\begin{aligned}
\left\|\delta \boldsymbol{e}^{n}\right\|^{2}+2 \delta t & \sum_{k=2}^{n}\left\|\boldsymbol{\nabla} \delta \widetilde{\boldsymbol{e}}^{k}\right\|_{\mathrm{L}^{2}}^{2}+\delta t^{2}\left\|\boldsymbol{\nabla} \delta \epsilon^{n}\right\|^{2} \\
& \leqslant \delta t \sum_{k=1}^{n-1} \delta t^{2}\left\|\boldsymbol{\nabla} \delta \epsilon^{k}\right\|^{2}+\left\|\delta \boldsymbol{e}^{1}\right\|^{2}+\delta t^{2}\left\|\boldsymbol{\nabla} \delta \epsilon^{1}\right\|^{2}+c T \delta t^{4} .
\end{aligned}
$$

Since the initialization of the scheme is such that $\boldsymbol{e}^{0}=0$ and $\epsilon^{0}=0$ and we have arbitrarily set $\widetilde{\boldsymbol{e}}^{0}=0, \delta \boldsymbol{e}^{1}=\boldsymbol{e}^{1}, \delta \widetilde{\boldsymbol{e}}^{1}=\widetilde{\boldsymbol{e}}^{1}, \delta \epsilon^{1}=\epsilon^{1}$ and $\psi^{1}=\bar{p}^{1}-\bar{p}^{0}$, so (3.7) written for $n=0$ yields:

$$
\left\|\delta \boldsymbol{e}^{1}\right\|^{2}+\left\|\delta \widetilde{\boldsymbol{e}}^{1}\right\|^{2}+2 \delta t\left\|\boldsymbol{\nabla} \delta \widetilde{\boldsymbol{e}}^{1}\right\|_{L^{2}}^{2}+\delta t^{2}\left\|\boldsymbol{\nabla} \delta \epsilon^{1}\right\|^{2}=\delta t^{2}\left\|\boldsymbol{\nabla}\left(\bar{p}^{1}-\bar{p}^{0}\right)\right\|^{2} \leqslant c \delta t^{4},
$$

and we conclude the proof by the discrete Gronwall lemma.

Remark 3.4 (A milder Regularity assumption) Assumption (3.11) is the discrete counterpart of an estimate for the second time-derivative of the pressure solution to the implicit scheme in $\mathrm{L}^{\infty}\left(0, T ; \mathrm{H}^{1}(\Omega)\right)$. Once again, proof of Lemma 3.5 shows that that a control in discrete $\mathrm{L}^{2}\left(0, T ; \mathrm{H}^{1}(\Omega)\right)$ norm would be sufficient, i.e.:

$$
\sum_{n=1}^{N-1} \delta t\left\|\boldsymbol{\nabla}\left(\bar{p}^{n+1}-2 \bar{p}^{n}+\bar{p}^{n-1}\right)\right\|^{2} \leqslant c \delta t^{4} .
$$

We finish by giving an error estimate for the pressure. To this purpose, we now need to make the following assumptions for the considered functional spaces:

- the pair $(\mathcal{V} \times \mathcal{Q})$ satisfies the so-called inf-sup condition:

$$
\forall q \in \mathcal{Q}, \quad \sup _{\boldsymbol{v} \in \mathcal{V}} \frac{(\boldsymbol{\nabla} q, \boldsymbol{v})}{\|\boldsymbol{\nabla} \boldsymbol{v}\|_{\mathrm{L}^{2}}} \geqslant \beta\|q\|_{\mathrm{L}^{2}}
$$

where $\beta$ is a given real number,

- the functions of $\mathcal{V}$ satisfy a Poincaré inequality, i.e. there exists a real number $c_{p}$ such that:

$$
\forall \boldsymbol{v} \in \mathcal{V}, \quad\|\boldsymbol{v}\| \leqslant c_{p}\|\nabla \boldsymbol{v}\|_{\mathrm{L}^{2}}
$$


Lemma 3.6 (ERror estimate for the PRESSURe) Let us suppose that Inequalities (3.5) and (3.11) hold. Then, under assumptions (3.14) and (3.15), there exists $c$ only depending on $\beta, c_{p}$ and the regularity of the solution to the implicit scheme such that:

$$
\sum_{n=0}^{N-1} \delta t\left\|\epsilon^{n}\right\|_{\mathrm{L}^{2}}^{2} \leqslant c \delta t^{2} .
$$

Proof. The first equation (3.6a) of the system controlling the splitting errors reads, for $0 \leqslant n<N$ and any $\boldsymbol{v} \in \mathcal{V}$ :

$$
\begin{aligned}
& \left(\boldsymbol{\nabla} \epsilon^{n}, \boldsymbol{v}\right)=-\frac{1}{\delta t}\left(\widetilde{\boldsymbol{e}}^{n+1}-\boldsymbol{e}^{n}, \boldsymbol{v}\right)-\left\langle\boldsymbol{\nabla} \widetilde{\boldsymbol{e}}^{n+1}, \boldsymbol{\nabla} \boldsymbol{v}\right\rangle+\left(\boldsymbol{\nabla}\left(\bar{p}^{n+1}-\bar{p}^{n}\right), \boldsymbol{v}\right) \\
& =-\frac{1}{\delta t}\left(\widetilde{\boldsymbol{e}}^{n+1}-\boldsymbol{e}^{n+1}, \boldsymbol{v}\right)-\frac{1}{\delta t}\left(\boldsymbol{e}^{n+1}-\boldsymbol{e}^{n}, \boldsymbol{v}\right)-\left\langle\boldsymbol{\nabla} \widetilde{\boldsymbol{e}}^{n+1}, \boldsymbol{\nabla} \boldsymbol{v}\right\rangle+\left(\boldsymbol{\nabla}\left(\bar{p}^{n+1}-\bar{p}^{n}\right), \boldsymbol{v}\right),
\end{aligned}
$$

so (3.14) and (3.15) yield:

$$
\left\|\epsilon^{n}\right\|_{L^{2}} \leqslant c_{1}\left(\frac{1}{\delta t}\left\|\widetilde{\boldsymbol{e}}^{n+1}-\boldsymbol{e}^{n+1}\right\|+\frac{1}{\delta t}\left\|\boldsymbol{e}^{n+1}-\boldsymbol{e}^{n}\right\|+\left\|\boldsymbol{\nabla} \widetilde{\boldsymbol{e}}^{n+1}\right\|_{\mathrm{L}^{2}}+\left\|\boldsymbol{\nabla}\left(\bar{p}^{n+1}-\bar{p}^{n}\right)\right\|\right),
$$

with $c_{1}$ only depending on $\beta$ and $c_{p}$. We thus obtain:

$$
\begin{aligned}
\left\|\epsilon^{n}\right\|_{\mathrm{L}^{2}}^{2} \leqslant 4 c_{1}^{2}\left(\frac{1}{\delta t^{2}}\left\|\widetilde{\boldsymbol{e}}^{n+1}-\boldsymbol{e}^{n+1}\right\|^{2}+\frac{1}{\delta t^{2}}\left\|\boldsymbol{e}^{n+1}-\boldsymbol{e}^{n}\right\|^{2}\right. \\
\left.+\left\|\nabla \widetilde{\boldsymbol{e}}^{n+1}\right\|_{\mathrm{L}^{2}}^{2}+\left\|\boldsymbol{\nabla}\left(\bar{p}^{n+1}-\bar{p}^{n}\right)\right\|^{2}\right),
\end{aligned}
$$

and we conclude by multiplying by $\delta t$ and summing over $n$, using (3.9) (which is a consequence of Lemma 3.5), Lemma 3.5, Lemma 3.2 and (3.5).

\subsection{Discrete variational setting and error estimates}

The aim of this section is to show that the considered scheme enters the formalism used in Section 3.1, and that the discrete spaces also satisfy the assumptions of Section 3.1, which yields a control of the splitting errors.

We begin with defining the discrete gradient operators. For the velocity, we use the usual definition, i.e. for $v \in \boldsymbol{V}$, we define $\boldsymbol{\nabla} \boldsymbol{v}$ as the function of $\mathrm{L}^{2}(\Omega)^{d \times d}$ equal to the gradient of $\boldsymbol{v}$ almost everywhere in $\Omega$ (namely, in the interior of the cells). Let us turn to pressure gradients. For $q \in Q$, we define $\boldsymbol{\nabla} q$ as the function of $\boldsymbol{V}$ defined by:

$$
\forall \sigma \in \mathcal{E}_{\mathrm{int}}, \sigma=K \mid L, \quad(\nabla q)_{\sigma}=\frac{|\sigma|}{\left|D_{\sigma}\right|}\left(q_{L}-q_{K}\right) \boldsymbol{n}_{K, \sigma}
$$

Note that, since we have supposed that $\boldsymbol{\nabla} \in \in \boldsymbol{V},(\boldsymbol{\nabla} q)_{\sigma}=0$ for any external face.

We now identify the abstract space $\mathcal{V}$ of Section 3.1 to $\boldsymbol{V}, \mathcal{Q}$ to $Q$ and, with the definition (3.16) of the pressure gradient, we have $\mathcal{X}=\boldsymbol{V}$. The discrete inner product on $\mathcal{X}$ (here $\boldsymbol{V}$ ) is chosen as:

$$
\forall \boldsymbol{v}, \boldsymbol{w} \in \boldsymbol{V}, \quad(\boldsymbol{v}, \boldsymbol{w})=\sum_{\sigma \in \mathcal{E}_{\mathrm{int}}}\left|D_{\sigma}\right| \boldsymbol{v}_{\sigma} \cdot \boldsymbol{w}_{\sigma}
$$


Note that, with this definition, the $\|\cdot\|$ and $\mathrm{L}^{2}$ norms are equivalent.

Combining the definition of the discrete pressure gradient (3.16) and the definition of the inner product (3.17), we get, for any $\boldsymbol{v} \in \boldsymbol{V}$ and $q \in Q$ :

$$
(\nabla q, \boldsymbol{v})=\sum_{\sigma \in \mathcal{E}_{\mathrm{int}}, \sigma=K \mid L}|\sigma|\left(q_{L}-q_{K}\right) \boldsymbol{v}_{\sigma} \cdot \boldsymbol{n}_{K, \sigma}=-\sum_{K \in \mathcal{M}} \int_{K} q \operatorname{div} \boldsymbol{v} \mathrm{d} \boldsymbol{x} .
$$

This relation has two consequences. First, it is now easy to see that the scheme written as (3.1) is indeed the scheme under consideration. Second, the inf-sup condition (3.14) is in fact the usual inf-sup condition for the Rannacher-Turek or Crouzeix-Raviart elements, and is thus satisfied.

Similarly, the (classical) definition of the gradient of velocities implies that the Poincaré inequality (3.15) also holds.

We now turn to the study of the properties of the inverse Stokes operator. To this purpose, we use the following lemmas. The proof of the first one may be found in $[16,3]$; the second one follows from trace lemmas obtained for general domains of $\mathbb{R}^{d}$ ([1, Theorem 3.10], or exploit Lemma A.1 in [7]), combined with the regularity assumption on the mesh.

Lemma 3.7 (Poincaré Inequality) For any convex domain $\omega$, for any function $\Phi \in \mathrm{H}^{1}(\omega)$ such that

$$
\int_{\omega} \Phi(x) d x=0
$$

we have:

$$
\|\Phi\|_{\mathrm{L}^{2}(\omega)} \leqslant \frac{\operatorname{diam}(\omega)}{\pi}\|\nabla \Phi\|_{\mathrm{L}^{2}(\omega)^{d}}
$$

Lemma 3.8 (A tRACE LEMma) Let $K$ be a cell of $\mathcal{M}, \sigma$ a face of $K$. Let $v$ be a function of $\mathrm{H}^{1}(K)$. Then there exists a real $c$ such that:

$$
\frac{1}{|\sigma|} \int_{\sigma} v^{2} \mathrm{~d} \gamma \leqslant \frac{c}{|K|}\left(\|v\|_{\mathrm{L}^{2}(K)}^{2}+h_{K}^{2}\|\nabla v\|_{\mathrm{L}^{2}(K)^{d}}^{2}\right),
$$

where $h_{K}$ stands for the diameter of $K$.

We also need to introduce a real number $c_{u}$ characterizing the quasi-uniformity of the mesh, defined by the fact that the following inequality holds:

$$
\max _{K \in \mathcal{M}, \sigma \in \mathcal{E}(K)} \frac{|\sigma|}{\left|D_{K, \sigma}\right|} \leqslant \frac{c_{u}}{h},
$$

where $h=\max _{K \in \mathcal{M}} h_{K}$.

The properties of the inverse Stokes operator are stated in the following lemma.

Lemma 3.9 Let $S^{-1}$ be the discrete inverse Stokes operator given by Definition 3.1. Then Property (S1) is satisfied. 
Let us suppose in addition that the continuous problem is regularizing, in the sense that, for any function $\underline{\boldsymbol{f}} \in \mathrm{L}^{2}(\Omega)^{d}$, the solution $(\underline{\boldsymbol{u}}, \underline{\zeta}) \in \mathrm{H}_{0}^{1}(\Omega)^{d} \times \mathrm{L}_{0}^{2}(\Omega)$ of the system:

$$
\begin{array}{ll}
\int_{\Omega} \boldsymbol{\nabla} \underline{\boldsymbol{u}}: \nabla \underline{\boldsymbol{v}} \mathrm{d} \boldsymbol{x}-\int_{\Omega} \underline{\zeta} \operatorname{div} \underline{\boldsymbol{v}} \mathrm{d} \boldsymbol{x}=\int_{\Omega} \underline{\boldsymbol{f}} \cdot \underline{\boldsymbol{v}} \mathrm{d} \boldsymbol{x}, & \\
\int_{\Omega} \underline{q} \operatorname{div} \underline{\boldsymbol{u}} \mathrm{d} \boldsymbol{x}=0, & \forall \underline{q} \in \mathrm{H}_{0}^{1}(\Omega)^{2}(\Omega),
\end{array}
$$

satisfies $\|\boldsymbol{\nabla} \underline{\zeta}\|_{\mathrm{L}^{2}(\Omega)^{d}} \leqslant c\|\boldsymbol{f}\|_{\mathrm{L}^{2}(\Omega)^{d}}$, with $c$ depending only on $\Omega$; this property holds if $\Omega$ is convex. Then assumption (S2) is satisfied, with a function $c(\alpha)$ depending in an increasing way on the real number $c_{u}$ of Equation (3.18) characterizing the quasi-uniformity of the mesh.

Proof. Let $\boldsymbol{v} \in \boldsymbol{V}$. We recall that $S^{-1} \boldsymbol{v}$ is defined by $S^{-1} \boldsymbol{v} \in \boldsymbol{V}$ and:

$$
\begin{array}{ll}
\left\langle\boldsymbol{\nabla} S^{-1} \boldsymbol{v}, \boldsymbol{\nabla} \boldsymbol{w}\right\rangle+(\boldsymbol{\nabla} \zeta, \boldsymbol{w})=(\boldsymbol{v}, \boldsymbol{w}), & \forall \boldsymbol{w} \in \boldsymbol{V}, \\
\left(\boldsymbol{\nabla} q, S^{-1} \boldsymbol{v}\right)=0, & \forall q \in Q,
\end{array}
$$

with $\zeta \in Q$.

Proof of (S1) - Let $\boldsymbol{w} \in \boldsymbol{V}$. Then, taking $S^{-1} \boldsymbol{w}$ as test function in (3.19a), we have:

$$
\left(\boldsymbol{v}, S^{-1} \boldsymbol{w}\right)=\left\langle\nabla S^{-1} \boldsymbol{v}, \boldsymbol{\nabla} S^{-1} \boldsymbol{w}\right\rangle+\left(\boldsymbol{\nabla} \zeta, S^{-1} \boldsymbol{w}\right),
$$

and thus, thanks to the fact that $S^{-1} \boldsymbol{w}$ is discretely divergence free (i.e. by (3.19b) written with $\boldsymbol{w}$ instead of $\boldsymbol{v}$ for the definition of $S^{-1} \boldsymbol{w}$ ):

$$
\left(\boldsymbol{v}, S^{-1} \boldsymbol{w}\right)=\left\langle\nabla S^{-1} \boldsymbol{v}, \nabla S^{-1} \boldsymbol{w}\right\rangle,
$$

which yields the symmetry and positivity property we are searching for.

Proof of (S2) - For the remainder of the proof, we denote by $c_{i}$ a real number only depending on $\Omega$ and, possibly, on the regularity of the mesh. Let us define by $\hat{\boldsymbol{v}}$ the function of $\boldsymbol{V}$ such that:

$$
\forall \boldsymbol{w} \in \boldsymbol{V}, \quad(\hat{\boldsymbol{v}}, \boldsymbol{w})=\int_{\Omega} \boldsymbol{v} \cdot \boldsymbol{w} \mathrm{d} \boldsymbol{x} .
$$

Thanks to the equivalence between the $\|\cdot\|$ and the $\mathrm{L}^{2}$-norm, we get that:

$$
\|\hat{\boldsymbol{v}}\|_{\mathrm{L}^{2}(\Omega)^{d}} \leqslant c_{1}\|\boldsymbol{v}\|_{\mathrm{L}^{2}(\Omega)^{d}} .
$$

Let us denote by $\left(\underline{S}^{-1} \hat{\boldsymbol{v}}, \underline{\zeta}\right)$ the solution of the continuous Stokes problem with $\hat{\boldsymbol{v}}$ as right-hand side, i.e. $\left(\underline{S}^{-1} \hat{\boldsymbol{v}}, \underline{\zeta}\right) \in \mathrm{H}_{0}^{1}(\Omega){ }^{d} \times \mathrm{L}_{0}^{2}(\Omega)$ such that:

$$
\begin{array}{ll}
\int_{\Omega} \underline{S}^{-1} \hat{\boldsymbol{v}}: \nabla \underline{\boldsymbol{w}} \mathrm{d} \boldsymbol{x}-\int_{\Omega} \underline{\zeta} \operatorname{div} \underline{\boldsymbol{w}} \mathrm{d} \boldsymbol{x}=\int_{\Omega} \hat{\boldsymbol{v}} \cdot \underline{\boldsymbol{w}} \mathrm{d} \boldsymbol{x}, & \forall \underline{\boldsymbol{w}} \in \mathrm{H}_{0}^{1}(\Omega)^{d}, \\
\int_{\Omega} \underline{q} \operatorname{div}\left(\underline{S}^{-1} \hat{\boldsymbol{v}}\right) \mathrm{d} \boldsymbol{x}=0, & \forall \underline{q} \in \mathrm{L}^{2}(\Omega) .
\end{array}
$$

Since we suppose that the Stokes problem is regularizing, we get:

$$
\|\boldsymbol{\nabla} \underline{\zeta}\|_{\mathrm{L}^{2}(\Omega)^{d}} \leqslant c_{2}\|\boldsymbol{v}\|_{\mathrm{L}^{2}(\Omega)^{d}} .
$$


In addition, System (3.19) is a standard approximation of the above continuous Stokes problem, so, by standard convergence results for the Rannacher-Turek or Crouzeix-Raviart element:

$$
\|\zeta-\underline{\zeta}\|_{\mathrm{L}^{2}(\Omega)} \leqslant c_{3} h\|\boldsymbol{v}\|_{\mathrm{L}^{2}(\Omega)^{d}} .
$$

Let $\pi_{h}$ be the projection operator from $\mathrm{L}^{2}(\Omega)$ onto $Q$ obtained as taking the mean value over each cell:

$$
\forall \underline{\xi} \in \mathrm{L}^{2}(\Omega), \forall K \in \mathcal{M}, \quad\left(\pi_{h} \underline{\xi}\right)_{K}=\frac{1}{|K|} \int_{K} \underline{\xi} \mathrm{d} \boldsymbol{x} .
$$

By a straightforward application of Lemma 3.7, this operator satisfies the following approximation property:

$$
\forall \underline{\xi} \in \mathrm{H}^{1}(\Omega), \quad\left\|\underline{\xi}-\pi_{h} \underline{\xi}\right\|_{\mathrm{L}^{2}(\Omega)} \leqslant c_{4} h\|\boldsymbol{\nabla} \underline{\xi}\|_{\mathrm{L}^{2}(\Omega)} .
$$

In addition, it satisfies the following stability property:

$$
\forall \underline{\xi} \in \mathrm{H}^{1}(\Omega), \quad\left\|\nabla \pi_{h} \underline{\xi}\right\| \leqslant c_{5}\|\nabla \underline{\xi}\|_{L^{2}(\Omega)^{d}} .
$$

Indeed:

$$
\begin{aligned}
& \left\|\nabla \pi_{h} \underline{\xi}\right\|^{2}=\sum_{\substack{\sigma \in \mathcal{E}_{\text {int }} \\
(\sigma=K \mid L)}} \frac{|\sigma|^{2}}{\left|D_{\sigma}\right|}\left[\frac{1}{|K|} \int_{K} \underline{\xi} \mathrm{d} \boldsymbol{x}-\frac{1}{|L|} \int_{L} \underline{\xi} \mathrm{d} \boldsymbol{x}\right]^{2} \\
& \quad \leqslant 2 \sum_{\substack{\sigma \in \mathcal{E}_{\text {int }} \\
(\sigma=K \mid L)}} \frac{|\sigma|^{2}}{\left|D_{\sigma}\right|}\left[\left(\frac{1}{|K|} \int_{K} \underline{\xi} \mathrm{d} \boldsymbol{x}-\frac{1}{|\sigma|} \int_{\sigma} \underline{\xi} \mathrm{d} \gamma\right)^{2}+\left(\frac{1}{|L|} \int_{L} \underline{\xi} \mathrm{d} \boldsymbol{x}-\frac{1}{|\sigma|} \int_{\sigma} \underline{\xi} \mathrm{d} \gamma\right)^{2}\right]
\end{aligned}
$$

Let $\underline{\xi}^{K}$ be the function defined over $K$ by:

$$
\underline{\xi}^{K}=\underline{\xi}-\frac{1}{|K|} \int_{K} \underline{\xi} \mathrm{d} \boldsymbol{x} .
$$

With this notation, for $\sigma \in \mathcal{E}_{\text {int }}, \sigma=K \mid L$, we get:

$$
T_{\sigma, K}=\left(\frac{1}{|K|} \int_{K} \underline{\xi} \mathrm{d} \boldsymbol{x}-\frac{1}{|\sigma|} \int_{\sigma} \underline{\xi} \mathrm{d} \gamma\right)^{2}=\left(\frac{1}{|\sigma|} \int_{\sigma} \underline{\xi}^{K} \mathrm{~d} \gamma\right)^{2} .
$$

By, successively, the Jensen inequality, the trace lemma 3.8 and the Poincaré inequality for zero mean-valued functions (Lemma 3.7):

$$
\begin{aligned}
T_{\sigma, K} \leqslant \frac{1}{|\sigma|} \int_{\sigma}\left(\underline{\xi}^{K}\right)^{2} \mathrm{~d} \gamma & \leqslant \frac{c_{6}}{|K|}\left[\left\|\underline{\xi}^{K}\right\|_{\mathrm{L}^{2}(K)}^{2}+h_{K}^{2}\|\boldsymbol{\nabla} \underline{\xi}\|_{\mathrm{L}^{2}(K)^{d}}^{2}\right] \\
& \leqslant \frac{c_{6}}{|K|}\left[\frac{h_{K}^{2}}{\pi^{2}}\|\boldsymbol{\nabla} \underline{\xi}\|_{\mathrm{L}^{2}(K)^{d}}^{2}+h_{K}^{2}\|\boldsymbol{\nabla} \underline{\xi}\|_{\mathrm{L}^{2}(K)^{d}}^{2}\right] \leqslant c_{7} \frac{h_{K}^{2}}{|K|}\|\boldsymbol{\nabla} \underline{\xi}\|_{\mathrm{L}^{2}(K)^{d}}^{2} .
\end{aligned}
$$

Returning to (3.23), we get:

$$
\left\|\nabla \pi_{h} \underline{\xi}\right\|^{2} \leqslant c_{8} \sum_{\substack{\sigma \in \mathcal{E}_{\text {int }} \\(\sigma=K \mid L)}} \frac{|\sigma|^{2}}{\left|D_{\sigma}\right|}\left[\frac{h_{K}^{2}}{|K|}\|\nabla \underline{\xi}\|_{\mathrm{L}_{2}(K)^{d}}^{2}+\frac{h_{L}^{2}}{|L|}\|\boldsymbol{\nabla} \underline{\xi}\|_{\mathrm{L}^{2}(L)^{d}}^{2}\right],
$$


which yields (3.22) invoking the regularity of the mesh.

We are now in position to provide an estimate for $\boldsymbol{\nabla} \zeta$. By an inverse inequality proven below, then by (3.20), (3.21) and (3.22), we have:

$$
\begin{aligned}
\|\nabla \zeta\| \leqslant\left\|\nabla\left(\zeta-\pi_{h} \underline{\zeta}\right)\right\|+\left\|\nabla \pi_{h} \underline{\zeta}\right\| \leqslant \frac{c_{10}}{h}\left\|\zeta-\pi_{h} \underline{\zeta}\right\|+\left\|\nabla \pi_{h} \underline{\zeta}\right\| \\
\leqslant \frac{c_{10}}{h}\left[\|\zeta-\underline{\zeta}\|+\left\|\underline{\zeta}-\pi_{h} \underline{\zeta}\right\|\right]+\left\|\nabla \pi_{h} \underline{\zeta}\right\| \leqslant c_{11}\|\boldsymbol{v}\| .
\end{aligned}
$$

Thus, for any $\boldsymbol{w} \in \boldsymbol{H}$, with $\boldsymbol{H}$ the space of discretely divergence-free functions, and $\alpha \in(0,1)$ :

$$
\begin{array}{r}
\left\langle\boldsymbol{\nabla} S^{-1} \boldsymbol{v}, \boldsymbol{\nabla} \boldsymbol{v}\right\rangle=\|\boldsymbol{v}\|^{2}-(\boldsymbol{\nabla} \zeta, \boldsymbol{v})=\|\boldsymbol{v}\|^{2}-(\boldsymbol{\nabla} \zeta, \boldsymbol{v}-\boldsymbol{w}) \geqslant\|\boldsymbol{v}\|^{2}-\|\boldsymbol{\nabla} \zeta\|\|\boldsymbol{u}-\boldsymbol{w}\| \\
\geqslant\|\boldsymbol{v}\|^{2}-c_{11}\|\boldsymbol{v}\|\|\boldsymbol{u}-\boldsymbol{w}\| \geqslant(1-\alpha)\|\boldsymbol{v}\|^{2}-\frac{c_{11}}{4 \alpha}\|\boldsymbol{u}-\boldsymbol{w}\|,
\end{array}
$$

which is the inequality we are searching for.

In the preceding proof, we have used the following lemma.

LEMma 3.10 (InVERSE INEQUALITY) The discrete pressure gradient operator defined by (3.16) and the discrete scalar product defined by (3.17) are such that the following inverse inequality is satisfied:

$$
\forall q \in Q, \quad\|\nabla q\| \leqslant \theta_{\mathcal{M}}\|q\|_{L^{2}(\Omega)} \quad \text { with } \theta_{\mathcal{M}}=\max _{K \in \mathcal{M}, \sigma \in \mathcal{E}(K)} \frac{|\sigma|}{\left|D_{K, \sigma}\right|} .
$$

Proof. Let $q \in Q$. By definition, we have:

$$
\|\boldsymbol{\nabla} q\|^{2}=\sum_{\substack{\sigma \in \mathcal{E}_{\text {int }} \\(\sigma=K \mid L)}} \frac{|\sigma|^{2}}{\left|D_{\sigma}\right|}\left(q_{K}-q_{L}\right)^{2} \leqslant 2 \sum_{\substack{\sigma \in \mathcal{E}_{\text {int }} \\(\sigma=K \mid L)}} \frac{|\sigma|^{2}}{\left|D_{\sigma}\right|}\left[\left(q_{K}^{2}+q_{L}^{2}\right] .\right.
$$

Reordering the summations, we get:

$$
\begin{aligned}
\|\nabla q\|^{2} \leqslant \sum_{K \in \mathcal{M}} q_{K}^{2} \sum_{\sigma=K \mid L} \frac{|\sigma|^{2}}{\left|D_{\sigma}\right|} & \leqslant \sum_{K \in \mathcal{M}} q_{K}^{2} \sum_{\sigma \in \mathcal{E}(K)} \frac{|\sigma|^{2}}{\left|D_{K, \sigma}\right|} \\
& \leqslant \theta_{\mathcal{M}}^{2} \sum_{K \in \mathcal{M}} q_{K}^{2} \sum_{\sigma \in \mathcal{E}(K)}\left|D_{K, \sigma}\right|,
\end{aligned}
$$

which concludes the proof, since $\sum_{\sigma \in \mathcal{E}(K)}\left|D_{K, \sigma}\right|=|K|$.

The application of the results of Section 3.1 now yields the following error bounds.

Theorem 3.2 (SPLitTing ERRor ESTIMATES)

Let $\left(\boldsymbol{u}^{n}, p^{n}\right)_{0 \leqslant n \leqslant N}$ be the solution to the projection scheme, $\left(\overline{\boldsymbol{u}}^{n}, \bar{p}^{n}\right)_{0 \leqslant n \leqslant N}$ be the solution to the implicit scheme (3.4), and the splitting errors be defined by:

$$
\boldsymbol{e}^{n}=\boldsymbol{u}^{n}-\overline{\boldsymbol{u}}^{n}, \quad \widetilde{\boldsymbol{e}}^{n}=\widetilde{\boldsymbol{u}}^{n}-\overline{\boldsymbol{u}}^{n}, \quad \epsilon^{n}=p^{n}-\bar{p}^{n},
$$

for $0 \leqslant n \leqslant N$. We suppose that we can set $e^{0}=0$ and $\epsilon^{0}=0$, for instance because the time step associated to $n=0$ does not correspond to the first one, and that the algorithm has been 
initialized with some (at least one) iterations of the implicit scheme; in addition, we arbitrarily set $\widetilde{\boldsymbol{e}}^{0}=0$, which is possible because $\widetilde{\boldsymbol{u}}^{0}$ never appears in the algorithm. Then we have the following error estimates.

1. Let us assume that the solution of the implicit scheme satisfies the time-regularity assumption $\left\|\boldsymbol{\nabla}\left(\bar{p}^{n+1}-\bar{p}^{n}\right)\right\| \leqslant c_{1} \delta t$, for $0 \leqslant n<N$. Then the velocity error satisfies the following first-order estimate:

$$
\left\|\boldsymbol{e}^{n}\right\|^{2}+2 \delta t \sum_{k=1}^{n}\left\|\nabla \widetilde{\boldsymbol{e}}^{k}\right\|_{\mathrm{L}^{2}}^{2} \leqslant c \delta t^{2},
$$

where $c$ only depends on $c_{1}$, the domain and the regularity of the mesh.

2. If we assume in addition that the solution of the implicit scheme is more regular, namely that $\left\|\boldsymbol{\nabla}\left(\bar{p}^{n+1}-2 \bar{p}^{n}+\bar{p}^{n-1}\right)\right\| \leqslant c_{2} \delta t^{2}$, for $0<n<N$, then the pressure error satisfies the following first-order estimate:

$$
\sum_{n=0}^{N-1} \delta t\left\|\epsilon^{n}\right\|_{\mathrm{L}^{2}}^{2} \leqslant c \delta t^{2}
$$

where $c$ only depends on $c_{1}, c_{2}$, the domain and the regularity of the mesh.

3. If, in addition, we suppose that the Stokes problem is regularizing in the sense of Lemma 3.9 , then the velocity error satisfies the following second-order estimate:

$$
\sum_{n=0}^{N} \delta t\left\|\widetilde{\boldsymbol{e}}^{n}\right\|^{2}+\sum_{n=0}^{N} \delta t\left\|\boldsymbol{e}^{n}\right\|^{2} \leqslant c \delta t^{4}
$$

where $c$ depends on $c_{1}, c_{2}$, the domain, the regularity of the mesh, and, in an increasing way on the real number $c_{u}$ of Equation (3.18) characterizing the quasi-uniformity of the mesh.

\section{Numerical tests}

In the following, we assess the convergence properties of the scheme through two examples: we begin with a case with Dirichlet boundary conditions prescribed on the whole boundary of $\Omega$ (Section 4.1), then we turn to a case with open boundary conditions (Section 4.2).

The computations presented here are performed with the ISIS code [14], built from the software component library PELICANS [17], both under development at IRSN and available as open-source softwares.

\subsection{A Stokes flow with Dirichlet boundary conditions}

For this test case, we assume that $\Omega$ is a circle of diameter equal to one and $\partial \Omega=\Gamma_{D}$, i.e. $\Gamma_{N}=\emptyset$. We calculate the forcing term $\underline{f}$ such that the exact velocity and pressure fields, $\underline{\boldsymbol{u}}$ and $\underline{p}$, are given by:

$$
\underline{\boldsymbol{u}}\left(\boldsymbol{x}_{1}, \boldsymbol{x}_{2}, t\right)=\left[\begin{array}{c}
\sin \left(\boldsymbol{x}_{1}\right) \sin \left(\boldsymbol{x}_{2}+t\right) \\
\cos \left(\boldsymbol{x}_{1}\right) \cos \left(\boldsymbol{x}_{2}+t\right)
\end{array}\right], \quad \underline{p}\left(\boldsymbol{x}_{1}, \boldsymbol{x}_{2}, t\right)=\cos \left(\boldsymbol{x}_{1}\right) \sin \left(\boldsymbol{x}_{2}+t\right) .
$$


The initial pressure and velocity are equal to zero and, on $\partial \Omega$, we prescribe non-homogeneous Dirichlet boundary conditions, i.e. $\underline{\boldsymbol{u}}=\underline{\boldsymbol{u}}_{D}, \underline{\boldsymbol{u}}_{D}$ being the trace of $\underline{\boldsymbol{u}}$ on $\partial \Omega$.

The spatial discretization relies on the Crouzeix-Raviart finite element, and we use three simplicial almost uniform meshes, with 3600,14400 and 57600 cells respectively.

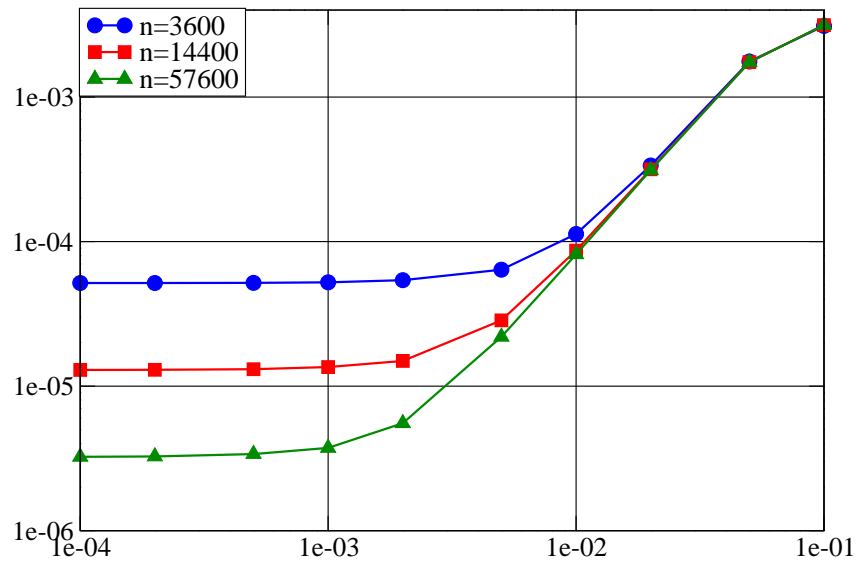

FIG. 1. Dirichlet case - Error $\left(\mathrm{L}^{2}\right.$-norm) for the velocity as a function of $\delta t$.

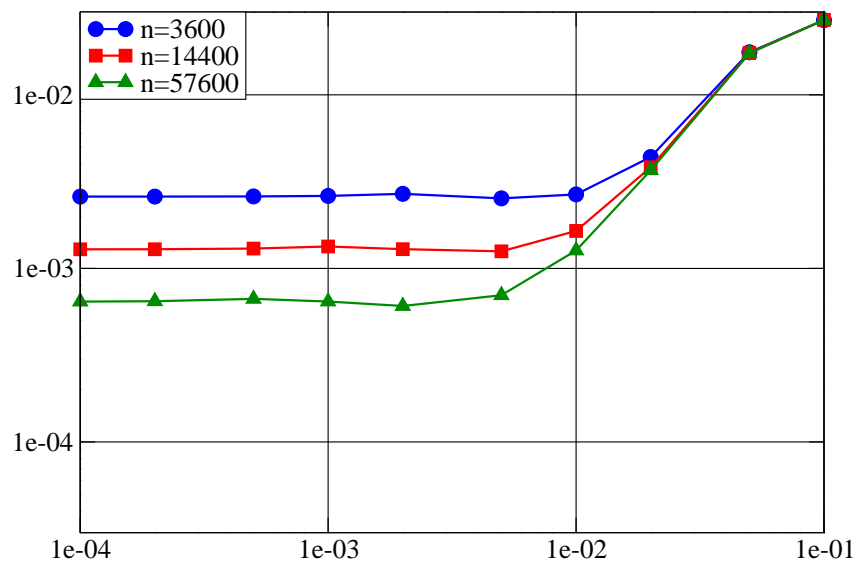

FIG. 2. Dirichlet case - Error $\left(\mathrm{L}^{2}\right.$-norm) for the pressure as a function of $\delta t$.

The numerical errors (measured in $\mathrm{L}^{2}$-norm) obtained at $t=1$, for the velocity and the 
pressure, as a function of the time step and for the various meshes, are plotted on Figures 1 and 2 respectively. The errors first decrease with the time step, then a plateau is reached, which corresponds to the residual error in space. The order of convergence in time is close to 2 for the velocity and 1 for the pressure; this may be surprising, since we use an only first-order backward Euler scheme, but, consistently with the error analysis given above, it is explained by the fact that the error is essentially due to the splitting. On the plateau, we observe a second-order convergence in space for the velocity and first-order for the pressure, that is the optimal order of convergence with our (low-degree) approximation.

As usual, Neumann boundary conditions prescribed in Equation (2.3) perturb the pressure field essentially near the boundary, as represented in Figure 3, obtained with $\delta t=10^{-4}$ and the finest mesh. Some errors also appear along some lines corresponding to a brutal variation of the size of the elements, resulting from the process used for the construction of the mesh.

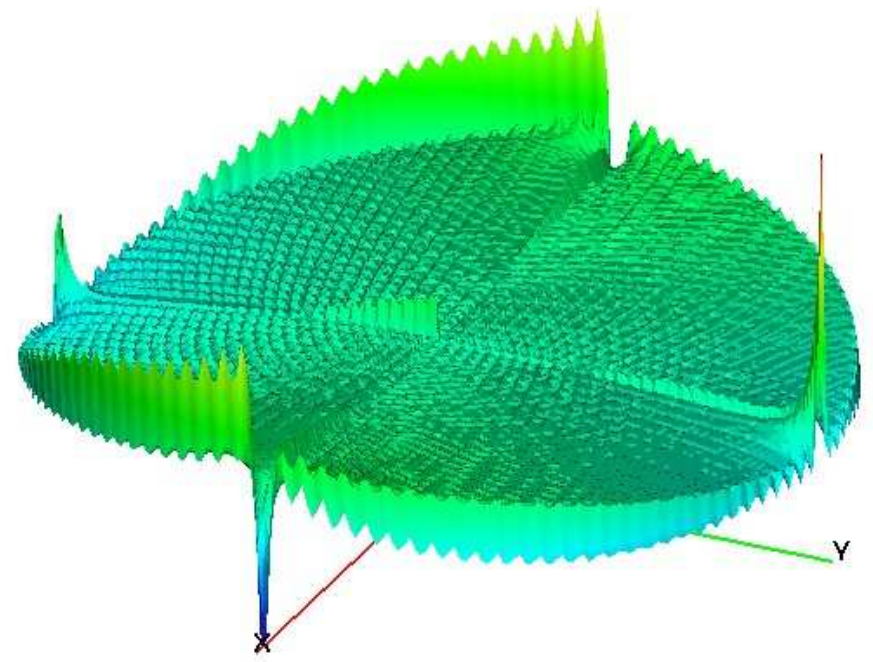

FIG. 3. Dirichlet case - Repartition of the pressure error ( $\mathrm{L}^{2}$-norm).

\subsection{Open boundary conditions}

In this test, the computational domain $\Omega$ is the unit square $[0,1]^{2}$ with $\Gamma_{N}$ equal to the vertical left side (and so $\Gamma_{D}=\partial \Omega \backslash \Gamma_{N}$ is equal to the three other sides). We calculate the forcing term $\boldsymbol{f}$ and choose the initial and boundary conditions in such a way that the exact velocity and pressure fields are the same as in the previous section. We obtain on $\Gamma_{N}$ :

$$
\boldsymbol{\nabla u} \cdot \boldsymbol{n}-p \boldsymbol{n}=0
$$

We plot on Figures 4 and 5 the numerical error as a function of the time step, measured in $\mathrm{L}^{2}$-norm and calculated at a fixed time, for $20 \times 20,40 \times 40$ and $80 \times 80$ structured uniform 
meshes. As previously, the errors first decrease with the time step to reach a plateau, which corresponds to the residual error in space. The order of convergence in time is once again close to 2 for the velocity and 1 for the pressure, still because the splitting error is predominant. On the plateau, we observe a second-order convergence in space for the velocity and first-order for the pressure, that is the optimal order of convergence with our (low-degree) approximation; by comparison, in [15], the authors observed for a Taylor-Hood (i.e. $Q_{2}-Q_{1}$ ) approximation a convergence rate of only 1 for the velocity and $1 / 2$ for the pressure, so, for the velocity, the present computations become more accurate already for the $80 \times 80$ mesh. Finally, on Figure 6 , we plot the error for the pressure in $\mathrm{L}^{\infty}$-norm; after a stagnation (at large $\delta t$ ), they show a convergence, which proves that the spurious homogeneous Dirichlet boundary condition for the pressure increment is indeed present for large time steps and relaxed for small ones.

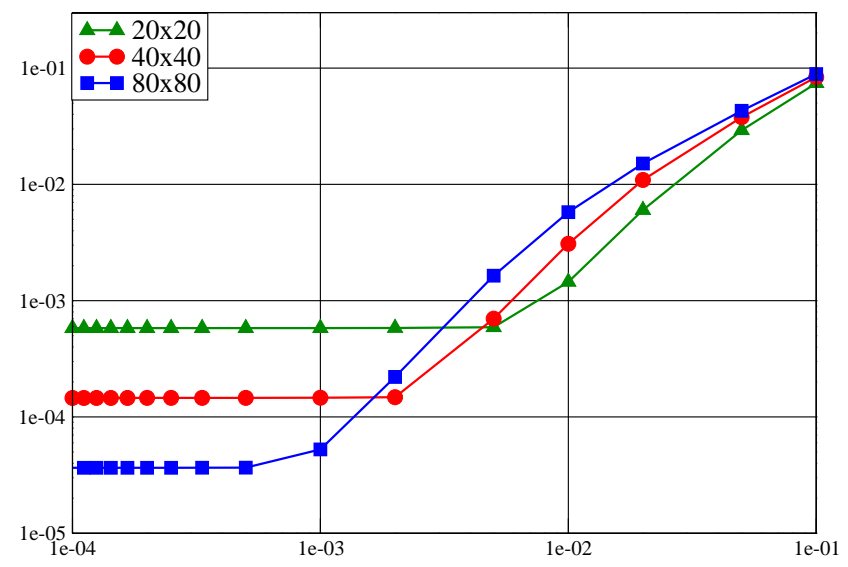

FIG. 4. Neumann case - Error for the velocity $\left(\mathrm{L}^{2}\right.$-norm) as a function of $\delta t$.

\section{REFERENCES}

[1] S. Agmon. Lectures on Elliptic Boundary Value Problems. AMS Chelsea Publishing, 2010.

[2] C. Amrouche and V. Girault. On the existence and regularity of the solution of Stokes problem in arbitrary dimension. Proc. Japan Acad., Série A, 67:171-175, 1991.

[3] M. Bebendorf. A note on the Poincaré inequality for convex domains. Journal for Analysis and its Applications, 22:751-756, 2003.

[4] L. Cattabriga. Su un problema al contorno relativo al sistemo di equazioni di Stokes. Rend. Sem. Mat. Univ. Padova, 31:308-340, 1961.

[5] A.J. Chorin. Numerical solution of the Navier-Stokes equations. Mathematics of Computation, 22:745$762,1968$.

[6] M. Crouzeix and P.A. Raviart. Conforming and nonconforming finite element methods for solving the stationary Stokes equations. RAIRO Série Rouge, 7:33-75, 1973.

[7] R. Eymard, R. Herbin, J.-C. Latché, and B. Piar. Convergence analysis of a locally stabilized collocated finite volume scheme for incompressible flows. Mathematical Modelling and Numerical Analysis, 


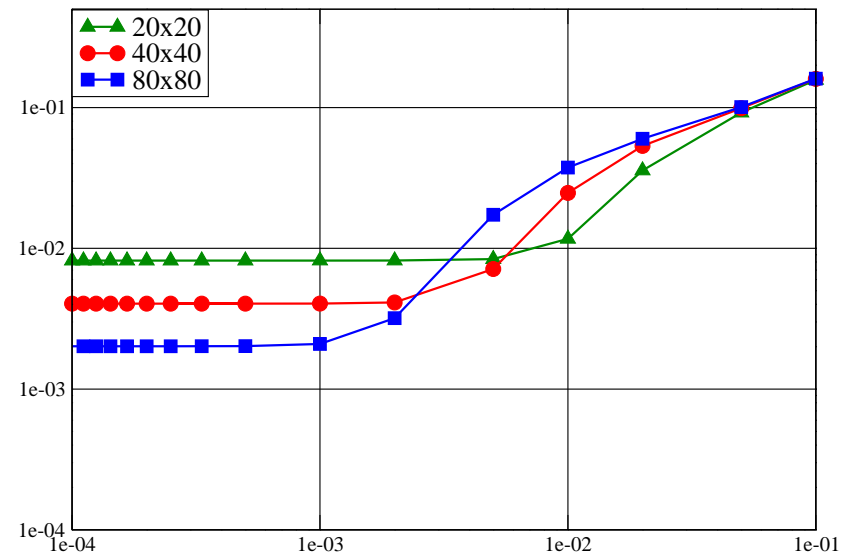

FIG. 5. Neumann case - Error for the pressure $\left(\mathrm{L}^{2}\right.$-norm) as a function of $\delta t$.

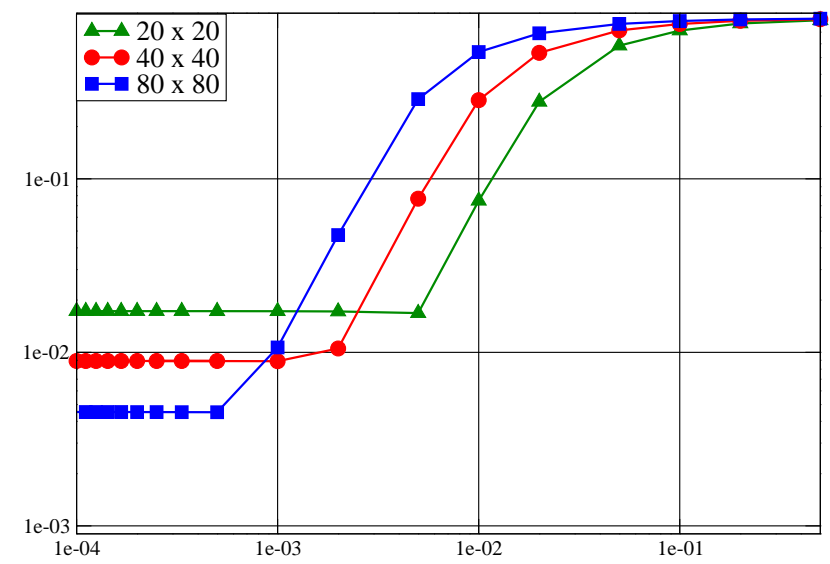

FIG. 6. Neumann case - Error for the pressure $\left(\mathrm{L}^{\infty}\right.$-norm) as a function of $\delta t$.

43:889-927, 2009.

[8] V. Girault and P.-A. Raviart. Finite Element Methods for Navier-Stokes Equations. Theory and Algorithms, volume 5 of Springer Series in Computational Mathematics. Springer-Verlag, 1986.

[9] K. Goda. A multistep technique with implicit difference schemes for calculating two- or threedimensional cavity flows. Journal of Computational Physics, 30:76-95, 1979.

[10] J.-L. Guermond. Some implementations of projection methods for Navier-Stokes equations. Mathematical Modelling and Numerical Analysis, 30:637-667, 1996.

[11] J.-L. Guermond. Un résultat de convergence d'ordre deux en temps pour l'approximation des équations de Navier-Stokes par une technique de projection incrémentale. Mathematical Modelling and 
Numerical Analysis, 33:169-189, 1999.

[12] J.-L. Guermond, P. Minev, and J. Shen. Error analysis of pressure-correction schemes for the timedependent Stokes equations with open boundary conditions. SIAM Journal on Numerical Analysis, 43:239-258, 2005.

[13] J.-L. Guermond and L. Quartapelle. On the approximation of the unsteady Navier-Stokes equations by finite element projection methods. Numerische Mathematik, 80:207-238, 1998.

[14] ISIS. A CFD computer code for the simulation of reactive turbulent flows. https://gforge.irsn.fr/gf/project/isis.

[15] M. Jobelin, C. Lapuerta, J.-C. Latché, Ph. Angot, and B. Piar. A finite element penalty-projection method for incompressible flows. Journal of Computational Physics, 217:502-518, 2006.

[16] L.E. Payne and H.F. Weinberger. An optimal Poincaré-inequality for convex domains. Archive for Rational Mechanics and Analysis, 5:286-292, 1960.

[17] PELICANS. Collaborative development environment. https://gforge.irsn.fr/gf/project/pelicans.

[18] A. Quarteroni and A. Valli. Numerical Approximation of Partial Differential Equations. Springer, 1994.

[19] R. Rannacher and S. Turek. Simple nonconforming quadrilateral Stokes element. Numerical Methods for Partial Differential Equations, 8:97-111, 1992.

[20] J. Shen. On error estimates of projection methods for Navier-Stokes equations: First-order schemes. SIAM Journal on Numerical Analysis, 29:57-77, 1992.

[21] J. Shen. Remarks on the pressure estimates for the projection methods. Numerische Mathematik, 67:513-520, 1994.

[22] J. Shen. On error estimates of projection methods for Navier-Stokes equations: Second-order schemes. Mathematics of Computation, 65:1039-1065, 1996.

[23] R. Temam. Sur l'approximation de la solution des équations de Navier-Stokes par la méthode des pas fractionnaires II. Arch. Rat. Mech. Anal., 33:377-385, 1969.

[24] J. van Kan. A second-order accurate pressure correction scheme for viscous incompressible flow. SIAM Journal on Scientific and Statistical Computing, 7:870-891, 1986. 